\title{
Reasoning About Knowledge of Unawareness Revisited* $^{*}$
}

\author{
Joseph Y. Halpern \\ Computer Science Department \\ Cornell University \\ Ithaca, NY, 14853, U.S.A. \\ halpern@cs.cornell.edu \\ Leandro C. Rêgo ${ }^{\dagger}$ \\ Statistics Department \\ Federal University of Pernambuco \\ Recife, PE, 50740-040, Brazil \\ leandro@de.ufpe.br
}

December 4, 2013

\footnotetext{
*A preliminary version of this work appears in the proceedings of the Conference on Theoretical Aspects of Rationality and Knowledge, 2009 (TARK09).

${ }^{\dagger}$ Corresponding author, phone: +55-81-2126-7438, fax: +55-81-2126-7438.
} 


\begin{abstract}
In earlier work (Halpern and Rêgo, 2009), we proposed a logic that extends the Logic of General Awareness of Fagin and Halpern (1988) by allowing quantification over primitive propositions. This makes it possible to express the fact that an agent knows that there are some facts of which he is unaware. In that logic, it is not possible to model an agent who is uncertain about whether he is aware of all formulas. To overcome this problem, we keep the syntax of the earlier paper, but allow models where, with each world, a possibly different language is associated. We provide a sound and complete axiomatization for this logic and show that, under natural assumptions, the quantifier-free fragment of the logic is characterized by exactly the same axioms as the logic of Heifetz, Meier, and Schipper (2008).
\end{abstract}

\title{
1 INTRODUCTION
}

Adding awareness to standard models of epistemic logic has been shown to be useful in describing many situations (see (Fagin and Halpern, 1988; Heifetz et al., 2006) for some examples). One of the best-known models of awareness is due to Fagin and Halpern (1988) (FH from now on). They add an awareness operator to the language, and associate with each world in a standard possible-worlds model of knowledge a set of formulas that each agent is aware of. They then say that an agent implicitly knows a formula $\varphi$ if $\varphi$ is true in all worlds that the agent considers possible (this is the traditional definition of knowledge, going back to Hintikka (1962)); an agent explicitly knows $\varphi$ if the agent implicitly knows $\varphi$ and is aware of $\varphi .^{1}$

In the economics literature, going back to the work of Modica and Rustichini $(1994 ; 1999)$ (MR from now on), a somewhat different approach is taken. A possibly different set $\mathcal{L}(s)$ of primitive propositions is associated with each world $s$. Intuitively, at world $s$, the agent is aware only of formulas that use the primitive propositions in $\mathcal{L}(s)$. A definition of knowledge is given in this framework, and the agent is said to be aware of $\varphi$ if, by definition, $K_{i} \varphi \vee K_{i} \neg K_{i} \varphi$ holds. Heifetz, Meier, and Schipper (2006; 2008) (HMS from now on), extend the ideas of MR to a multiagent setting. This extension is nontrivial, requiring lattices of state spaces, with projection functions

\footnotetext{
${ }^{1}$ Interestingly, Hintikka (1962) distinguished between a weak and strong sense of knowledge, where, roughly speaking, weak knowledge of $\varphi$ only requires the agent to have the information that $\varphi$ was true, while strong knowledge requires, in addition, justification for the information. As Sillari (2008a) points out, we could view explicit knowledge as strong knowledge and implicit knowledge as weak knowledge, if we interpret awareness as justification (although this is not the spirit in which it is typically interpreted).
} 
between them. As we showed in earlier work (Halpern, 2001; Halpern and Rêgo, 2008), the work of MR and HMS can be seen as a special case of the FH approach, where two assumptions are made on awareness: awareness is generated by primitive propositions, that is, an agent is aware of a formula iff he is aware of all primitive propositions occurring in it, and agents know what they are aware of (so that each agent is aware of the same formulas in all worlds that he consider possible).

As we pointed out in (Halpern and Rêgo, 2009) (referred to as HR from now on), if awareness is generated by primitive propositions, then it is impossible for an agent to explicitly know that he is unaware of a specific fact. Nevertheless, an agent may well be aware that there are relevant facts that he is unaware of. For example, primary-care physicians know that specialists are aware of things that could improve a patient's treatment that they are not aware of; investors know that investment fund companies may be aware of issues involving the financial market that could result in higher profits that they are not aware of. It thus becomes of interest to model knowledge of lack of awareness. HR does this by extending the syntax of the FH approach to allow quantification, making it possible to say that an agent knows that there exists a formula of which the agent is unaware. A complete axiomatization is provided for the resulting logic. Unfortunately, the logic has a significant problem if we assume the standard properties of knowledge and awareness: it is impossible for an agent to be uncertain about whether he is aware of all formulas. For example, suppose that an oncologist missed the latest international conference on oncology, and is uncertain as to whether any new treatment options were presented. Thus, the oncologist is uncertain about whether he is aware of all treatment options. This situation could not be modeled using the logic of HR, but can be modeled using the approach presented in this paper. Note that while the agent is aware of the concept of a treatment for cancer, he may be unaware of specific treatments. For example, the idea of a treatment that involves radiation may not even be on his radar screen, so to speak.

In this paper, we deal with this problem by considering the same language as in $\mathrm{HR},{ }^{2}$ but using the idea of MR that there is a different language associated with each world. As we show, this slight change makes it possible for an agent to be uncertain about whether he is aware of all formulas, while still being aware of exactly the same formulas in all worlds he considers possible. We provide a natural complete axiomatization for the resulting

\footnotetext{
${ }^{2}$ In this language, quantification can be used to express the fact that an agent (explicitly) knows that he is not aware of all formulas.
} 
logic. Interestingly, implicit knowledge in this logic acts much like explicit knowledge in the original FH framework, if we take "awareness of $\varphi$ " to mean $K_{i}(\varphi \vee \neg \varphi)$; intuitively, this formula is true if agent $i$ knows all the relevant concepts in $\varphi$; that is, if all the primitive propositions in $\varphi$ are part of the language at all worlds that $i$ considers possible. Under minimal assumptions, $K_{i}(\varphi \vee \neg \varphi)$ is shown to be equivalent to $K_{i} \varphi \vee K_{i} \neg K_{i} \varphi$ : in fact, the quantifier-free fragment of the logic that just uses the $K_{i}$ operator is shown to be characterized by exactly the same axioms as the HMS approach, and awareness can be defined the same way. Thus, we can capture the essence of the HMS approach without requiring the lattice structures of HMS, and instead associating a possibly different language with each world. By adding quantification to the language, we can in addition reason about knowledge of lack of awareness; it is not clear how to do this in the HMS approach. It turns out that, once we allow the possibility of different languages associated with different states, the gap between implicit knowledge and explicit knowledge narrows significantly. To understand this better, and to compare our framework carefully to the MR/HMS framework, we consider a language that includes both implicit and explicit knowledge, even though an agent reasons only about what he explicitly knows. In addition, under minimal assumptions, we show that we can define awareness using the implicit knowledge operator, and get equivalence of implicit and explicit knowledge.

Board and Chung (2009) independently pointed out the problem of the HR model and proposed the solution of allowing different languages at different worlds. They also consider a model of awareness with quantification, but they use first-order modal logic, so their quantification is over domain elements. Moreover, they take awareness with respect to domain elements, not formulas; that is, agents are (un)aware of objects (i.e., domain elements), not formulas. They also allow different domains at different worlds; more precisely, they allow an agent to have a subjective view of what the set of objects is at each world. Recent results of Board, Chung, and Schipper (2011) suggest that, in a sense, the Board and Chung approach is equally expressive as the HMS approach. Given the results of this paper, it should also be as expressive as our approach. Which approach lends itself more naturally to modeling applications of interest remains to be seen. While the examples of this paper are easily modeled with unawareness of formulas, there may be others that are more easily modeled using unawareness of objects.

Sillari (2008b), in independent work, uses much the same approach as Board and Chung (2009).That is, he has a first-order logic of awareness, where the quantification and awareness is with respect to domain elements; 
he also allows for different subjective domains at each world. He goes further by using what is called neighborhood semantics, also called Montague-Scott structures (Fagin et al., 1995). As is well known, neighborhood semantics provide a more general approach for modeling knowledge than the standard Kripke structures used here (and in most papers in computer science and economics). For example, they have been used to deal with the logical omniscience problem Fagin et al. (1995). But this greater flexibility comes at a price. Because it does not model knowledge in terms of possible worlds, but rather, in a sense, just provides a list of events that the agent knows, neighborhood semantics seem like a less natural way of modeling knowledge than Kripke structures (which is perhaps why it is used far less often in the literature). Neighborhood semantics and awareness are both ways of dealing with what has been called the logical omniscience problem: the fact that in the standard approaches, agents know all tautologies and know all the logical consequences of their knowledge. While the combination does give some greater generality, it does not seem necessary for most applications.

Agotnes and Alechina (2007) consider a restricted version of the HR logic, where we can reason only about certain types of unawareness (specifically, the only types of quantified statements allowed are ones that talk about whether an agent $i$ is aware of all formulas, and whether an agent $i$ is aware of everything that another agent $j$ is aware of). This restriction is still expressive enough to capture all the motivating examples considered in HR, while having significantly lower complexity (it is decidable, whereas the HR logic is undecidable). Unfortunately, the problem in the HR logic already occurs in the restricted version considered by Agotnes and Alechina. We could modify the Agotnes-Alechina logic along the lines of our modification here; we suspect that the improved complexity results would still obtain, although we have not checked.

Walker (2011) further restricts the logic so that the only types of quantified statements allowed are ones about whether an agent $i$ is aware of all formulas. In this restricted logic, he provides yet another solution to the problem in the HR logic. His solution has the advantage of not requiring different languages at different worlds. On the other hand, it is not clear how to extend his solution to a richer language. Moreover, in this logic an agent is allowed to have false beliefs about formulas that involve $\forall x A x$, but not for other type of formula.

The rest of the paper is organized as follows. In Section 2, we review the HR model of knowledge of unawareness. In Section 3, we present our new logic and axiomatize it in Section 4. In Section 4, we compare our logic with that of HMS and discuss awareness more generally. All proofs are left 
to the appendix.

\section{THE HR MODEL}

In this section, we briefly review the relevant results of (Halpern and Rêgo, 2009). The syntax of the logic is as follows: given a set $\{1, \ldots, n\}$ of agents, formulas are formed by starting with a countably infinite set $\Phi=\{p, q, \ldots\}$ of primitive propositions and a countably infinite set $\mathcal{X}$ of variables, and then closing off under conjunction $(\wedge)$, negation $(\neg)$, the modal operators $K_{i}, A_{i}, X_{i}, i=1, \ldots, n$. We also allow for quantification, so that if $\varphi$ is a formula, then so is $\forall x \varphi$. The domain of quantification is a subset of the set of formulas; see below for further discussion. Let $\mathcal{L}_{n}^{\forall, K, X, A}(\Phi, \mathcal{X})$ denote this language and let $\mathcal{L}_{n}^{K, X, A}(\Phi)$ be the subset of formulas that do not mention quantification or variables. As usual, we define $\varphi \vee \psi, \varphi \Rightarrow \psi$, and $\exists x \varphi$ as abbreviations of $\neg(\neg \varphi \wedge \neg \psi), \neg \varphi \vee \psi$, and $\neg \forall x \neg \varphi$, respectively. The intended interpretation of $A_{i} \varphi$ is " $i$ is aware of $\varphi$ ".

Essentially as in first-order logic, we can define inductively what it means for a variable $x$ to be free in a formula $\varphi$. Intuitively, an occurrence of a variable is free in a formula if it is not bound by a quantifier. A formula that contains no free variables is called a sentence. We are ultimately interested in sentences. If $\psi$ is a formula, let $\varphi[x / \psi]$ denote the formula that results by replacing all free occurrences of the variable $x$ in $\varphi$ by $\psi$. (If there is no free occurrence of $x$ in $\varphi$, then $\varphi[x / \psi]=\varphi$.) In quantified modal logic, the quantifiers are typically taken to range over propositions (intuitively, sets of worlds), but this does not work in our setting because awareness is syntactic; when we write, for example, $\forall x A_{i} x$, we essentially mean that $A_{i} \varphi$ holds for all formulas $\varphi$. However, there is another subtlety. If we define $\forall x \varphi$ to be true if $\varphi[x / \psi]$ is true for all formulas $\psi$, then there are problems giving semantics to a formula such as $\varphi=\forall x(x)$, since $\varphi[x / \varphi]=\varphi$. We avoid these difficulties by taking the domain of quantification to be the quantifier-free sentences, or more precisely, the formulas in $\mathcal{L}_{n}^{K, X, A}(\Phi)$. (See (Halpern and Rêgo, 2009) for further discussion.) $)^{3}$

We give semantics to sentences in $\mathcal{L}_{n}^{\forall, K, X, A}(\Phi, \mathcal{X})$ in awareness structures. A tuple $M=\left(S, \pi, \mathcal{K}_{1}, \ldots, \mathcal{K}_{n}, \mathcal{A}_{1}, \ldots, \mathcal{A}_{n}\right)$ is an awareness structure

\footnotetext{
${ }^{3}$ We remark that Feinberg (2011) has recently generalized our approach by replacing the $\forall$ in the syntax by a family of quantifiers $\forall^{m}$, for $m=0,1,2, \ldots$ Let $\mathcal{L}_{n}^{K, X, A, 0}=\mathcal{L}_{n}^{K, X, A}$, the quantifier-free formulas, and let $\mathcal{L}_{n}^{K, X, A, m}$ consist of all formulas that mention only the quantifiers $\forall^{m^{\prime}}$ for $m^{\prime}<m$. Feinberg takes the domain of quantification of $\forall^{m}$ to consist of all the formulas in $\mathcal{L}_{n}^{K, X, A, m}$; that is $\forall^{m} x \varphi$ is true at a world $s$ iff $\varphi[x / \psi]$ is true at $s$ for all formulas $\psi \in \mathcal{L}_{n}^{K, X, A, m}$.
} 
for $n$ agents (over $\Phi$ ) if $S$ is a set of worlds, $\pi: S \times \Phi \rightarrow\{$ true, false $\}$ is an interpretation that determines which primitive propositions are true at each world, $\mathcal{K}_{i}$ is a binary relation on $S$ for each agent $i=1, \ldots, n$, and $\mathcal{A}_{i}$ is a function associating a set of sentences with each world in $S$, for $i=1, \ldots, n$. Intuitively, if $(s, t) \in \mathcal{K}_{i}$, then agent $i$ considers world $t$ possible at world $s$, while $\mathcal{A}_{i}(s)$ is the set of sentences that agent $i$ is aware of at world $s$. We are often interested in awareness structures where the $\mathcal{K}_{i}$ relations satisfy some properties of interest, such as reflexivity, transitivity, or the Euclidean property (if $(s, t),(s, u) \in \mathcal{K}_{i}$, then $\left.(t, u) \in \mathcal{K}_{i}\right)$. It is well known that these properties of the relation correspond to properties of knowledge of interest (see Theorem 2.1 and the following discussion). We often abuse notation and define $\mathcal{K}_{i}(s)=\left\{t:(s, t) \in \mathcal{K}_{i}\right\}$, thus writing $t \in \mathcal{K}_{i}(s)$ rather than $(s, t) \in \mathcal{K}_{i}$. This notation allows us to view a binary relation $\mathcal{K}_{i}$ on $S$ as a possibility correspondence, that is, a function from $S$ to $2^{S}$. (The use of possibility correspondences is more standard in the economics literature than binary relations, but they are clearly essentially equivalent.)

Semantics is given to sentences in $\mathcal{L}_{n}^{\forall, K, X, A}(\Phi, \mathcal{X})$ by induction on the number of quantifiers, with a subinduction on the length of the sentence. Truth for primitive propositions, for $\neg$, and for $\wedge$ is defined in the usual way. The other cases are defined as follows: ${ }^{4}$

$$
\begin{aligned}
& (M, s) \models K_{i} \varphi \text { if }(M, t) \models \varphi \text { for all } t \in \mathcal{K}_{i}(s) \\
& (M, s) \models A_{i} \varphi \text { if } \varphi \in \mathcal{A}_{i}(s) \\
& (M, s) \models X_{i} \varphi \text { if }(M, s) \models A_{i} \varphi \text { and }(M, s) \models K_{i} \varphi \\
& (M, s) \models \forall x \varphi \text { if }(M, s) \models \varphi[x / \psi], \text { for all } \psi \in \mathcal{L}_{n}^{K, X, A}(\Phi) .
\end{aligned}
$$

In the HR model, as in standard epistemic logics, the intended interpretation of $K_{i} \varphi$ is that agent $i$ considers possible only worlds where $\varphi$ is true, while, as in the FH model, $X_{i} \varphi$ is true if, in addition to $\varphi$ being true in all worlds that $i$ considers possible, $i$ is aware of $\varphi$.

There are two standard restrictions on agents' awareness that capture the assumptions typically made in the game-theoretic literature (Modica and Rustichini, 1999; Heifetz et al., 2006, 2008). We describe these here in terms of the awareness function, and then characterize them axiomatically.

- Awareness is generated by primitive propositions (agpp) if, for all agents $i, \varphi \in \mathcal{A}_{i}(s)$ iff all the primitive propositions that appear in $\varphi$ are in $\mathcal{A}_{i}(s) \cap \Phi$.

\footnotetext{
${ }^{4} \mathrm{HR}$ gives semantics to arbitrary formulas, including formulas with free variables. This requires using valuations that give meaning to free variables. By restricting to sentences, which is all we are ultimately interested in, we are able to dispense with valuations here, and thus simplify the presentation of the semantics.
} 
- Agents know what they are aware of (ka) if, for all agents $i$ and all worlds $s, t$ such that $(s, t) \in \mathcal{K}_{i}$ we have that $\mathcal{A}_{i}(s)=\mathcal{A}_{i}(t)$.

To be able to relate our results better to those in the economics literature, we restrict attention in this paper to structures that satisfy agpp and $k a$. If $C$ is a (possibly empty) subset of $\{r, t, e\}$, then $\mathcal{M}_{n}^{C}(\Phi, \mathcal{X})$ is the set of all awareness structures such that awareness satisfies agpp and $k a$ and the possibility correspondence is reflexive $(r)$, transitive $(t)$, and Euclidean $(e)$ if these properties are in $C$.

A sentence $\varphi \in \mathcal{L}_{n}^{\forall, K, X, A}(\Phi, \mathcal{X})$ is said to be valid in awareness structure $M$, written $M \models \varphi$, if $(M, s) \not \models \neg \varphi$ for all $s \in S$. We remark that this notion is called weak validity in (Halpern and Rêgo, 2008). For the semantics we are considering here, weak validity is equivalent to the standard notion of validity, where a formula is valid in an awareness structure if it is true at all worlds in that structure. However, in the next section, we modify the semantics to allow some formulas to be undefined at some worlds; with this change, the two notions do not coincide. As we use weak validity in the next section, we use the same definition here for the sake of uniformity. A sentence is valid in a class $\mathcal{M}$ of awareness structures, written $\mathcal{M} \models \varphi$, if it is valid for all awareness structures in $\mathcal{M}$, that is, if $M \models \varphi$ for all $M \in \mathcal{M}$.

An axiom system AX consists of a collection of axioms and inference rules. An axiom is a formula, and an inference rule has the form "from $\varphi_{1}, \ldots, \varphi_{k}$ infer $\psi$," where $\varphi_{1}, \ldots, \varphi_{k}, \psi$ are formulas. A formula $\varphi$ is provable in (or a theorem of) $\mathrm{AX}$, denoted $\mathrm{AX} \vdash \varphi$, if there is a sequence of formulas such that the last one is $\varphi$, and each one is either an axiom or follows from previous formulas in the sequence by an application of an inference rule.

In (Halpern and Rêgo, 2009), we gave sound and complete axiomatizations for both the language $\mathcal{L}_{n}^{\forall, K, X, A}(\Phi, \mathcal{X})$ and the language $\mathcal{L}_{n}^{\forall, X, A}(\Phi, \mathcal{X})$, which does not mention the implicit knowledge operator $K_{i}$ (and the quantification is thus only over sentences in $\mathcal{L}_{n}^{X, A}(\Phi)$ ). The latter language is arguably more natural (since agents do not have access to the implicit knowledge modeled by $K_{i}$ ), but some issues become clearer when considering both. In particular, considering both allows us to analyze the relationship between $K_{i}$ and $X_{i}$ more carefully, and to compare our approach to the work of Heifetz, Meier, and Schiper and of Modica and Rustichinni.

We start by describing axioms for the language $\mathcal{L}_{n}^{\forall, K, X, A}(\Phi, \mathcal{X})$, and then describe how they are modified to deal with $\mathcal{L}_{n}^{\forall, X, A}(\Phi, \mathcal{X})$. Given a formula $\varphi$, let $\Phi(\varphi)$ be the set of primitive propositions in $\Phi$ that occur in $\varphi$.

Prop. All substitution instances of valid formulas of propositional logic. 
AGPP. $A_{i} \varphi \Leftrightarrow \wedge_{p \in \Phi(\varphi)} A_{i} p .^{5}$

KA. $A_{i} \varphi \Rightarrow K_{i} A_{i} \varphi$

NKA. $\neg A_{i} \varphi \Rightarrow K_{i} \neg A_{i} \varphi$

K. $\left(K_{i} \varphi \wedge K_{i}(\varphi \Rightarrow \psi)\right) \Rightarrow K_{i} \psi$.

T. $K_{i} \varphi \Rightarrow \varphi$.

4. $K_{i} \varphi \Rightarrow K_{i} K_{i} \varphi$.

5. $\neg K_{i} \varphi \Rightarrow K_{i} \neg K_{i} \varphi$.

A0. $X_{i} \varphi \Leftrightarrow K_{i} \varphi \wedge A_{i} \varphi$.

$1_{\forall} \cdot \forall x \varphi \Rightarrow \varphi[x / \psi]$ if $\psi$ is a quantifier-free sentence.

$\mathrm{K}_{\forall} \cdot \forall x(\varphi \Rightarrow \psi) \Rightarrow(\forall x \varphi \Rightarrow \forall x \psi)$.

$\mathrm{N}_{\forall} \cdot \varphi \Rightarrow \forall x \varphi$ if $x$ is not free in $\varphi$.

Barcan. $\forall x K_{i} \varphi \Rightarrow K_{i} \forall x \varphi$.

MP. From $\varphi$ and $\varphi \Rightarrow \psi$ infer $\psi$ (modus ponens).

$\operatorname{Gen}_{K}$. From $\varphi$ infer $K_{i} \varphi$.

Gen $_{\forall}$. If $q$ is a primitive proposition, then from $\varphi$ infer $\forall x \varphi[q / x]$.

Axioms Prop, K, T, 4, 5 and inference rules MP and $\mathrm{Gen}_{K}$ are standard in epistemic logics. A0 captures the relationship between explicit knowledge, implicit knowledge and awareness, and just says that explicit knowledge is equivalent to the combination of implicit knowledge and awareness; this is just the FH definition of explicit knowledge. Axioms $1_{\forall}, \mathrm{K}_{\forall}, \mathrm{N}_{\forall}$ and inference rule $\mathrm{Gen}_{\forall}$ capture standard properties of universal quantification (and have exact analogues in first-order logic, where the quantification is over objects). For example, $1_{\forall}$ says that if a universally quantified formula is true, then so is each instance of it. (Bull, 1969; Engelhardt et al., 1998; Fine, 1970;

\footnotetext{
${ }^{5}$ As usual, the empty conjunction is taken to be the vacuously true formula true, so that $A_{i} \varphi$ is vacuously true if no primitive propositions occur in $\varphi$. We remark that in the conference version of HR, an apparently weaker version of AGPP called weak generation of awareness by primitive propositions is used. However, this is shown in HR to be equivalent to AGPP if the agent is aware of at least one primitive proposition, so AGPP is used in the final version of $\mathrm{HR}$, and we use it here as well.
} 
Kaplan, 1970; Kripke, 1959). ${ }^{6}$ The Barcan axiom, which is well-known in first-order modal logic, captures the relationship between quantification and $K_{i}$. It says that if agent $i$ knows $\varphi[x / \psi]$ for each quantifier-free formula $\psi$, then he knows $\forall x \varphi$. The converse to the Barcan formula holds as well, although we do not need it for the complete axiomatization. Axiom AGPP captures the key property of awareness being generated by primitive propositions; axioms KA and NKA capture the properties of agents knowing which formulas they are and are not aware of. Specifically, KA says that an agent knows what he is aware of; NKA says that he knows what he is not aware of. Let $\mathrm{AX}^{K, X, A, \forall}$ be the axiom system consisting of all the axioms and inference rules in Prop, AGPP, KA, NKA, K, A0, $1_{\forall}, \mathrm{K}_{\forall}, \mathrm{N}_{\forall}$, Barcan, MP, $\left.\operatorname{Gen}_{K}, \operatorname{Gen}_{\forall}\right\}$.

The language $\mathcal{L}_{n}^{\forall, X, A}$ without the modal operators $K_{i}$ has an axiomatization that is similar in spirit. Let $\mathrm{K}_{X}, \mathrm{~T}_{X}, 4_{X}, \mathrm{XA}$, and $\operatorname{Barcan}_{X}$ be the axioms that result by replacing the $K_{i}$ in $\mathrm{K}, \mathrm{T}, 4$, KA, and Barcan, respectively, by $X_{i}$. Let $5_{X}$ and $\operatorname{Gen}_{X}$ be the axioms that result from adding awareness to 5 and $\operatorname{Gen}_{K}$ :

$5_{X} \cdot\left(\neg X_{i} \varphi \wedge A_{i} \varphi\right) \Rightarrow X_{i} \neg X_{i} \varphi$.

$\operatorname{Gen}_{X}$. From $\varphi$ infer $A_{i} \varphi \Rightarrow X_{i} \varphi$.

The analogue of axiom NKA written in terms of $X_{i}, \neg A_{i} \varphi \Rightarrow X_{i} \neg A_{i} \varphi$, is not valid. An agent can never explicitly know what he is not aware of. Indeed, $\neg A_{i} \varphi \Rightarrow \neg X_{i} \neg A_{i} \varphi$ is valid. To get completeness in models where agents know what they are aware of, we need the following axiom, which can be viewed as a weakening of NKA:

$\mathrm{FA}_{X} \cdot \neg \forall x A_{i} x \Rightarrow X_{i} \neg \forall x A_{i} x$.

$\mathrm{FA}_{X}$ says that if an agent is not aware of all formulas, then he explicitly knows that. This may seem to be an unreasonable axiom. If an agent in fact is not aware of all formulas, why should he explicitly know this? It seems reasonable that he could mistakenly believe that he is aware of everything, even though he is not. Nevertheless, it is not hard to show that this formula is valid in the models considered by Halpern and Rêgo (2009), and is not valid in the models that we consider in this paper.

\footnotetext{
${ }^{6}$ Since we gave semantics not just to sentences, but also to formulas with free variables in (Halpern and Rêgo, 2009), we were able to use a simpler version of Gen $\forall$ that applies to arbitrary formulas: from $\varphi$ infer $\forall x \varphi$. Note that all the other axioms and inference rules apply without change to formulas as well as sentences.
} 
Finally, consider the following weakening of A0, which captures the relationship between explicit knowledge and awareness to the extent that we can talk about it without having $K_{i}$ in the language:

$\mathrm{A} 0_{X} \cdot X_{i} \varphi \Rightarrow A_{i} \varphi$

Let $\mathrm{AX}^{X, A, \forall}$ be the axiom system consisting of all the the axioms and inference rules in $\left\{\right.$ Prop, AGPP, XA, FA ${ }_{X}, \mathrm{~K}_{X}, \mathrm{~A} 0_{X}, 1_{\forall}, \mathrm{K}_{\forall}, \mathrm{N}_{\forall}, \operatorname{Barcan}_{X}$, MP, Gen $\left.X, \operatorname{Gen}_{\forall}\right\}$. The following result shows that the semantic properties $r, t, e$ are captured by the axioms $\mathrm{T}, 4$, and 5 , respectively in the language $\mathcal{L}_{n}^{\forall, K, X, A}$; similarly, these same properties are captured by $\mathrm{T}_{X}, 4_{X}$, and $5_{X}$ in the language $\mathcal{L}_{n}^{\forall, X, A}$.

Theorem 2.1 (Halpern and Rêgo, 2009) If $\mathcal{C}$ (resp., $\mathcal{C}_{X}$ ) is a (possibly empty) subset of $\{\mathrm{T}, 4,5\}$ (resp., $\left\{\mathrm{T}_{\mathrm{X}}, 4_{\mathrm{X}}, 5_{\mathrm{X}}\right\}$ ) and if $C$ is the corresponding subset of $\{r, t, e\}$ then $\mathrm{AX}^{K, X, A, \forall} \cup \mathcal{C}$ (resp., $\mathrm{AX}^{X, A, \forall} \cup \mathcal{C}_{X}$ ) is a sound and complete axiomatization of the sentences in $\mathcal{L}_{n}^{\forall, K, X, A}(\Phi, \mathcal{X})$ (resp. $\mathcal{L}_{n}^{\forall, X, A}(\Phi, \mathcal{X})$ ) with respect to $\mathcal{M}_{n}^{C}(\Phi, \mathcal{X}) .^{7}$

Consider the formula

$$
\psi=\neg X_{i} \neg \forall x A_{i} x \wedge \neg X_{i} \forall x A_{i} x .
$$

The formula $\psi$ says that agent $i$ considers it possible that she is aware of all formulas and also considers it possible that she is not aware of all formulas. It is not hard to show $\psi$ is not satisfiable in any structure in $\mathcal{M}(\Phi, \mathcal{X})$, so $\neg \psi$ is valid in awareness structures in $\mathcal{M}(\Phi, \mathcal{X})$. However, in many situations, it seems reasonable that an agent may be uncertain about whether there

\footnotetext{
${ }^{7}$ We remark that when we say "completeness" here and elsewhere in the paper, as usual, we mean, as usual, that every formula that is valid is provable. There is a stronger notion of completeness, called strong completeness. An axiom system $A X$ is strongly complete if, for every subset $\Psi$ of formulas, if $\varphi$ is valid in every structure $M$ such all the formulas in $\Psi$ are true at every state of $M$, then $\varphi$ is provable from $A X$ together with $\Psi$ (that is, all the formulas in $\Psi$ are taken as axioms). Strong completeness does not hold. Indeed, we cannot hope to provide a strongly complete axiomatization for our logic, since it is not even compact: there is an infinite set of formulas, each subset of which is satisfiable, although the infinite set is not. This follows because of the presence of the universal quantifier. Consider the infinite set $\Psi$ of formulas consisting of $A_{i} \varphi$ for all $\varphi \in \mathcal{L}_{n}^{K, X, A}$ and $\neg \forall x A_{i} x$. Clearly every finite subset of $\Psi$ is satisfiable, although the infinite set $\Psi$ is not. Since $\Psi$ is not satisfiable, all formulas are trivially valid in every structure where every formula in $\Psi$ is satisfiable (since there are no such structures). But because proofs are finitary, it is not hard to show that it is not the case that all formulas are provable from the result of adding $\Psi$ to any sound axiom system; indeed, the primitive proposition $p$ will not be provable.
} 
are formulas he is unaware of. In the next section, we show that a slight modification of the HR approach, using ideas of MR, allows this, while still maintaining the desirable properties of the HR approach.

\section{THE NEW MODEL}

We keep the syntax of Section 2, but, following MR, we allow different languages to be associated with different worlds. Define an extended awareness structure for $n$ agents (over $\Phi$ ) to be a tuple $M=\left(S, \mathcal{L}, \pi, \mathcal{K}_{1}, \ldots\right.$, $\left.\mathcal{K}_{n}, \mathcal{A}_{1}, \ldots, \mathcal{A}_{n}\right)$, where $M=\left(S, \pi, \mathcal{K}_{1}, \ldots, \mathcal{K}_{n}, \mathcal{A}_{1}, \ldots, \mathcal{A}_{n}\right)$ is an awareness structure and $\mathcal{L}$ maps worlds in $S$ to nonempty subsets of $\Phi$. Intuitively, $\mathcal{L}_{n}^{\forall, K, X, A}(\mathcal{L}(s), \mathcal{X})$ is the language associated with world $s$. We require that $\mathcal{A}_{i}(s) \subseteq \mathcal{L}_{n}^{\forall, K, X, A}(\mathcal{L}(s), \mathcal{X})$, so that an agent can be aware only of sentences that are in the language of the current world. Thus, in this model, associated with each world $s \in S$, there is an objective language $\mathcal{L}(s)$ that is sufficient to describe all relevant objective events in $s$; a subjective subset $\mathcal{A}_{i}(s)$ of $\mathcal{L}_{n}^{\forall, K, X, A}(\mathcal{L}(s), \mathcal{X})$, which characterizes the events in $s$ that $i$ is aware of; and a subjective description $\mathcal{K}_{i}(s)$ of the worlds that agent $i$ considers possible. We still want to require that agpp and $k a$; this means that if $(s, t) \in \mathcal{K}_{i}$, then $\mathcal{A}_{i}(s) \subseteq \mathcal{L}_{n}^{\forall, K, X, A}(\mathcal{L}(t), \mathcal{X})$. But $\mathcal{L}(t)$ may well include primitive propositions that the agent is not aware of at $s$. It may at first seem strange that an agent considers possible a world whose language includes formulas of which he is not aware. ${ }^{8}$ But, in the context of knowledge of lack awareness, there is an easy explanation for this: the fact that $\mathcal{A}_{i}(s)$ is a strict subset of the sentences in $\mathcal{L}_{n}^{\forall, K, X, A}(\mathcal{L}(t), \mathcal{X})$ is just our way of modeling that the agent considers it possible that there are formulas of which he is unaware; he can even "name" or "label" these formulas, although he may not understand what the names refer to. If the agent considers possible a world $t$ where $\mathcal{A}_{i}(s)$ consists of every sentence in $\mathcal{L}_{n}^{\forall, K, X, A}(\mathcal{L}(t), \mathcal{X})$, then the agent considers it possible that he is aware of all formulas. The formula $\psi$ defined in (1) is satisfied at a world $s$ where agent $i$ considers possible a world $t_{1}$ such that $\mathcal{A}_{i}(s)$ consists of all sentences in $\mathcal{L}_{n}^{\forall, K, X, A}\left(\mathcal{L}\left(t_{1}\right), \mathcal{X}\right)$ and a world $t_{2}$ such that $\mathcal{A}_{i}(s)$ does not contain some sentence in $\mathcal{L}_{n}^{\forall, K, X, A}\left(\mathcal{L}\left(t_{2}\right), \mathcal{X}\right)$.

What this suggests is that, informally, the new model allows for "levels" of unawareness. While there may be some concepts that agent $i$ is unaware of and not even realize are "out there", there may be other concepts that $i$ is unaware of where $i$ is aware of the "gap" in his awareness. The former

\footnotetext{
${ }^{8}$ Note that, in general, this happens in the HR approach too, even though there we require that $\mathcal{L}(s)=\mathcal{L}(t)$.
} 
situation can be modeled by a formula $p$ that agent $i$ is unaware of at a world $s$ such that $p$ is not in the language at any world that $i$ considers possible; the latter situation can be captured by having $p$ in the language at all worlds that $i$ considers possible. We can have intermediate cases as well, where $i$ is uncertain about whether there is a concept that he is unaware of (so that $p$ would be in the language at some worlds that $i$ considers possible, and not in the language at other worlds that $i$ considers possible). Roughly speaking, although $i$ cannot name the concepts that he is unaware of (if he could, he would be aware of them), he may have "labels" for concepts he is unaware of. For example, if $i$ is aware that there is a formula that he and $j$ are not aware of, but $k$ is aware of, and another formula that he and $k$ are aware of but $j$ is not aware of, then $i$ will need labels for these two distinct concepts, although he is aware of neither one. While, from $i$ 's point of view, these are just labels, a world $s$ will "flesh out" these labels and give them a concrete interpretation.

In this model, it is perfectly reasonable for $i$ to consider possible a world $t$ where neither he nor $j$ is aware of some formula $p$ (that is, $p \notin \mathcal{A}_{i}(t) \cup \mathcal{A}_{j}(t)$ ). For example, in the oncology example, $i$ might consider it possible that there is a specific oncology treatment $p$ that neither he nor $j$ is aware of. Of course, $p$ does not have an specific meaning; $p$ is just a label that $i$ uses to describe the treatment. But he can consider it possible that he and $j$ are unaware of the same treatment. We can similarly describe worlds where agent 1 considers it possible that agents 2 and 3 are aware of the same formulas, although both are aware of formulas that he (1) is not aware of, and other more complicated relationships between the awareness of agents. See Section 4 for further discussion of awareness of unawareness in this setting.

The truth relation is defined for formulas in $\mathcal{L}_{n}^{\forall, K, X, A}(\Phi, \mathcal{X})$ just as in Section 2, except that for a formula $\varphi$ to be true at a world $s$, we also require that $\varphi \in \mathcal{L}_{n}^{\forall, K, X, A}(\mathcal{L}(s), \mathcal{X})$, so we just add this condition everywhere. Our approach here is similar to that of MR. Intuitively, if $\varphi \notin \mathcal{L}_{n}^{\forall, K, X, A}(\mathcal{L}(s), \mathcal{X})$, then the truth value of $\varphi$ at $s$ is undefined. We do not have a three-valued logic, with an "undefined" truth value; instead, we take both $\varphi$ and $\neg \varphi$ to be false at $s$. We can essentially identify a formula $\varphi$ such that $\varphi$ and $\neg \varphi$ are both false at $s$ as a formula that is undefined at $s$. Thus, for example,

- $(M, s) \models p$ if $p \in \mathcal{L}(s)$ and $\pi(s, p)=$ true;

- $(M, s) \models \neg \varphi$ if $\varphi \in \mathcal{L}_{n}^{\forall, K, X, A}(\mathcal{L}(s), \mathcal{X})$ and $(M, s) \forall \models \varphi$.

- $(M, s) \models \forall x \varphi$ if $\varphi \in \mathcal{L}_{n}^{\forall, K, X, A}(\mathcal{L}(s), \mathcal{X})$ and 


$$
(M, s) \models \varphi[x / \psi] \text { for all } \psi \in \mathcal{L}_{n}^{K, X, A}(\mathcal{L}(s)) .
$$

We leave it to the reader to make the obvious changes to the remaining clauses. It is worth emphasizing that our interpretation of $K_{i} \varphi$ and $X_{i} \varphi$ remains exactly as in the HR model; in particular, $K_{i} \varphi$ holds iff agent $i$ considers possible only worlds where $\varphi$ is true, which implies that the language at every world considered possible by $i$ is rich enough to express $\varphi$.

If $C$ is a (possibly empty) subset of $\{r, t, e\}$, then $\mathcal{N}_{n}^{C}(\Phi, \mathcal{X})$ is the set of all extended awareness structures such that awareness satisfies agpp and $k a$ and the possibility correspondence is reflexive, transitive, and Euclidean if these properties are in $C$. We say that a formula $\varphi$ is valid in a class $\mathcal{N}$ of extended awareness structures if, for all extended awareness structures $M \in \mathcal{N}$ and worlds $s$ such that $\Phi(\varphi) \subseteq \mathcal{L}(s),(M, s) \models \varphi$. (This is essentially the notion of weak validity defined in (Halpern and Rêgo, 2008).)

\section{AXIOMATIZATION}

In this section, we provide a sound and complete axiomatization of the logics described in the previous section. It turns out to be easier to start with the language $\mathcal{L}_{n}^{\forall, X, A}(\Phi, \mathcal{X})$. All the axioms and inference rules of $\mathrm{AX}^{X, A, \forall}$ continue to be sound in extended awareness structures, except for $\operatorname{Barcan}_{X}$ and $\mathrm{FA}_{X}$. In a world $s$ where $\mathcal{L}(s)=\{p\}$ and agent 1 is aware of $p$, it is easy to see that $\forall x X_{i} A_{i} x$ holds. But if agent 1 considers possible a world $t$ such that $\mathcal{L}(t)=\{p, q\}$, it is easy to see that $X_{i} \forall x A_{i} x$ does not hold at $s$. Similarly, if in world $t$, agent 1 considers $s$ possible, then $\neg \forall x A_{i} x$ holds at $t$, but $X_{i} \neg \forall x A_{i} x$ does not. Thus, Barcan $_{X}$ does not hold at $s$, and $\mathrm{FA}_{X}$ does not hold at $t$. We instead use the following variants of $\operatorname{Barcan}_{X}$ and $\mathrm{FA}_{X}$, which are sound in this framework:

$\operatorname{Barcan}_{X}^{*} \cdot\left(A_{i}(\forall x \varphi) \wedge \forall x\left(A_{i} x \Rightarrow X_{i} \varphi\right)\right) \Rightarrow X_{i}\left(\forall x A_{i} x \Rightarrow \forall x \varphi\right)$.

$\mathrm{FA}_{X}^{*} . \forall x \neg A_{i} x \Rightarrow X_{i} \forall x \neg A_{i} x$.

Barcan $_{X}^{*}$ avoids the problem with $\operatorname{Barcan}_{X}$ mentioned above. Essentially, it relativizes $\operatorname{Barcan}_{X}$ to awareness. Thus, in the conclusion, rather than requiring that $\forall x \varphi$ be true in all worlds that the agent considers possible, it requires only that $\forall x \varphi$ be true in those worlds where the agent is aware of all formulas. And in the antecedent, rather than requiring that the agent (explicitly) know $\varphi[x / \psi]$ for all formulas $\psi$, the agent is required to know $\varphi[x / \psi]$ only if the agent is aware of $\psi$. There is an additional technical requirement in the antecedent that the agent be aware of the formula $\forall x \varphi$. 
$\mathrm{FA}_{X}^{*}$ seems much more reasonable that $\mathrm{FA}_{X}$. It says that if the agent is not aware of any formulas, then he explicitly knows this. It does not seem implausible that an agent should know that he is not aware of anything $\left(\forall x \neg A_{i} x\right)$, although he may not know that he is not aware of all formulas $\left(\neg \forall x A_{i} x\right)$. In any case, we clearly need to weaken $\mathrm{FA}_{X}$ if we do not want the formula $\psi$ in (1) to hold.

Let $\mathrm{AX}_{e}^{X, A, \forall}$ be the result of replacing $\mathrm{FA}_{X}$ and $\operatorname{Barcan}_{X}$ in $\mathrm{AX}^{X, A, \forall}$ by FA $_{X}^{*}$ and Barcan $_{X}^{*}$ (the $e$ here stands for "extended").

Theorem 4.1 If $\mathcal{C}_{X}$ is a (possibly empty) subset of $\left\{\mathrm{T}_{\mathrm{X}}, 4_{\mathrm{X}}, 5_{\mathrm{X}}\right\}$ and $C$ is the corresponding subset of $\{r, t, e\}$, then $\mathrm{AX}_{e}^{X, A, \forall} \cup \mathcal{C}_{X}$ is a sound and complete axiomatization of the language $\mathcal{L}_{n}^{\forall, X, A}(\Phi, \mathcal{X})$ with respect to $\mathcal{N}_{n}^{C}(\Phi, \mathcal{X})$.

The completeness proof is similar in spirit to that of HR, with some additional complications arising from the interaction between quantification and the fact that different languages are associated with different worlds. What is surprisingly difficult in this case is soundness, specifically, for MP. For suppose that $M$ is a structure in $\mathcal{N}_{n}(\Phi, \mathcal{X})$ such that neither $\neg \varphi$ nor $\neg(\varphi \Rightarrow \psi)$ are true at any world in $M$. We want to show that $\neg \psi$ is not true at any world in $M$. This is easy to show if $\Phi(\psi) \subset \Phi(\varphi)$. For if $s$ is a world such that $\Phi(\psi) \subseteq \mathcal{L}(s)$, it must be the case that both $\varphi$ and $\varphi \Rightarrow \psi$ are true at $s$, and hence so is $\psi$. However, if $\varphi$ has some primitive propositions that are not in $\psi$, it is a priori possible that $\neg \psi$ holds at a world where neither $\varphi$ nor $\varphi \Rightarrow \psi$ is defined. Indeed, this can happen if $\Phi$ is finite. For example, if $\Phi=\{p, q\}$, then it is easy to construct a structure $M \in \mathcal{N}_{n}(\Phi, X)$ where both $A_{i} p \wedge A_{i} q$ and $\left(A_{i} p \wedge A_{i} q\right) \Rightarrow \forall x A_{i} x$ are never false, but $\forall x A_{i} x$ is false at some world in $M$. As we show, this cannot happen if $\Phi$ is infinite. This in turn involves proving a general substitution property: if $\varphi$ is valid and $\psi$ is a quantifier-free sentence, then $\varphi[q / \psi]$ is valid. This substitution property also fails if $\Phi$ is finite. The fact that $\Phi$ is infinite guarantees that we can find a "fresh" primitive proposition, that is not mentioned in the formulas that we are considering. ${ }^{9}$ See the appendix for details.

Using different languages has a greater impact on the axioms for $K_{i}$ than it does for $X_{i}$. Since $K_{i}$ is affected by the language $\mathcal{L}(t)$ in the worlds $t$ that $i$ considers possible, and $X_{i}$ is affected in much the same way by the set $\mathcal{A}_{i}(t)$ of formulas that $i$ is aware of in the worlds $t$ that $i$ considers possible, in the new model, the properties of $K_{i}$ and $X_{i}$ are much more similar than in our earlier work.

\footnotetext{
${ }^{9}$ There are other cases where restricting to a finite set of primitive propositions results in extra axioms; see, for example, Fagin et al. (1992).
} 
For example, as we would expect, Barcan does not hold, for essentially the same reason that $\operatorname{Barcan}_{X}$ does not hold. More interestingly, NKA, 5 , and $\operatorname{Gen}_{K}$ do not hold either. For example, if $\neg K_{i} p$ is true at a world $s$ because $p \notin \mathcal{L}(t)$ for some world $t$ that $i$ considers possible at $s$, then $K_{i} \neg K_{i} p$ will not hold at $s$, even if the $\mathcal{K}_{i}$ relation is an equivalence relation. Indeed, the properties of $K_{i}$ in this framework become quite close to the properties of the explicit knowledge operator $X_{i}$ in the original FH framework, provided we define the appropriate variant of awareness.

Let $A_{i}^{*}(\varphi)$ be an abbreviation for the formula $K_{i}(\varphi \vee \neg \varphi)$. Intuitively, the formula $A_{i}^{*}(\varphi)$ captures the property that agent $i$ considers possible only worlds whose language is rich enough to describe $\varphi$. Note that, in general, $A_{i}^{*}(\varphi)$ does not imply $A_{i}(\varphi)$. It is possible for agent $i$ to consider possible only worlds whose language can express $\varphi$ without being aware of $\varphi$. Agent $i$ would then explicitly know that there is a formula that he is not aware of.

Let $\mathrm{AGPP}^{*}, \mathrm{XA}^{*}, \mathrm{~A}^{*}, 5^{*}, \mathrm{Barcan}^{*}, \mathrm{FA}^{*}$, and $\mathrm{Gen}^{*}$ be the result of replacing $X_{i}$ by $K_{i}$ and $A_{i}$ by $A_{i}^{*}$ in AGPP, XA, A $0_{X}, 5_{X}, \operatorname{Barcan}_{X}^{*}, \mathrm{FA}_{X}^{*}$, and $\operatorname{Gen}_{X}$, respectively. It is easy to see that AGPP*, A0*, and Gen* are valid in extended awareness structures; $\mathrm{XA}^{*}, 5^{*}$, Barcan*, and $\mathrm{FA}^{*}$ are not. For example, suppose that $p$ is defined in all worlds that agent $i$ considers possible at $s$, so that $A_{i}^{*} p$ holds at $s$. If there is some world $t$ that agent $i$ considers possible at $s$ and a world $u$ that agent $i$ considers possible at $t$ where $p$ is not defined, then $A_{i}^{*} p$ does not hold at $t$, so $K_{i} A_{i}^{*} p$ does not hold at $s$. It is easy to show that $\mathrm{XA}^{*}$ holds if the $\mathcal{K}_{i}$ relation is transitive. Similar arguments show that $5^{*}$, Barcan*, and $\mathrm{FA}^{*}$ do not hold in general, but are valid if $\mathcal{K}_{i}$ is Euclidean and (in the case of Barcan* and $\mathrm{FA}^{*}$ ) reflexive. We summarize these observations in the following proposition:

Proposition 4.1 (a) $X A^{*}$ is valid in $\mathcal{N}_{n}^{t}(\Phi, \mathcal{X})$.

(b) Barcan* is valid in $\mathcal{N}_{n}^{r, e}(\Phi, \mathcal{X})$.

(c) $F A^{*}$ is valid in $\mathcal{N}_{n}^{r, e}(\Phi, \mathcal{X})$.

(d) $5^{*}$ is valid in $\mathcal{N}_{n}^{e}(\Phi, \mathcal{X})$.

In light of Proposition 4.1, for ease of exposition, we restrict attention for the rest of this section to structures in $\mathcal{N}_{n}^{r, t, e}(\Phi, \mathcal{X})$. Assuming that the possibility relation is an equivalence relation is standard when modeling knowledge in any case. Let $\mathrm{AX}_{e}^{K, X, A, A^{*}, \forall}$ be the result of replacing $\mathrm{Gen}_{K}$ and Barcan in $\mathrm{AX}^{K, X, A, \forall}$ by Gen* and Barcan*, respectively, and adding the axioms $\mathrm{AGPP}^{*}, \mathrm{~A}^{*}$, and $\mathrm{FA}^{*}$ for reasoning about $A_{i}^{*}$. (We do not need the axiom $\mathrm{XA}^{*}$; it follows from 4 in transitive structures.) Let $\mathrm{AX}_{e}^{K, A^{*}, \forall}$ consist 
of the axioms in $\mathrm{AX}_{e}^{K, X, A, A^{*}, \forall}$ except for those that mention $X_{i}$ or $A_{i}$; that is, $\mathrm{AX}_{e}^{K, A^{*}, \forall}=\mathrm{AX}_{e}^{K, X, A, A^{*}, \forall}-\{\mathrm{AGPP}, \mathrm{KA}, \mathrm{NKA}, \mathrm{A} 0\}$. Note that $\mathrm{AX}_{e}^{K, A^{*}, \forall}$ is the result of replacing $X_{i}$ by $K_{i}$ and $A_{i}$ by $A_{i}^{*}$ in $\mathrm{AX}_{e}^{X, A, \forall}$ (except that the analogue of $\mathrm{XA}$ is not needed). Finally, let $\mathrm{AX}_{e}^{K, A^{*}}$ consist of the axioms and rules in $\mathrm{AX}_{e}^{K, A^{*}, \forall}$ except for the ones that mention quantification; that is, $\mathrm{AX}_{e}^{K, A^{*}}=\left\{\right.$ Prop, $\left.\mathrm{AGPP}^{*}, \mathrm{~K}, \mathrm{Gen}^{*}, \mathrm{~A} 0^{*}\right\}$. We use $\mathrm{AX}_{e}^{K, A^{*}}$ to compare our results to those of HMS.

Theorem 4.2 (a) $\mathrm{AX}_{e}^{K, A^{*}} \cup\left\{\mathrm{T}, 4,5^{*}\right\}$ is a sound and complete axiomatization of $\mathcal{L}_{n}^{K}(\Phi)$ with respect to $\mathcal{N}_{n}^{r, t, e}(\Phi)$.

(b) $\mathrm{AX}_{e}^{K, A^{*}, \forall} \cup\left\{\mathrm{T}, 4,5^{*}\right\}$ is a sound and complete axiomatization of the sentences in $\mathcal{L}_{n}^{\forall, K}(\Phi, \mathcal{X})$ with respect to $\mathcal{N}_{n}^{r, t, e}(\Phi, \mathcal{X})$.

(c) $\mathrm{AX}_{e}^{K, X, A, A^{*}, \forall} \cup\left\{\mathrm{T}, 4,5^{*}\right\}$ is a sound and complete axiomatization of the sentences in $\mathcal{L}_{n}^{\forall, K, X, A}(\Phi, \mathcal{X})$ with respect to $\mathcal{N}_{n}^{r, e, t}(\Phi, \mathcal{X})$.

Since, as we observed above, $\mathrm{AX}_{e}^{K, A^{*}, \forall}$ is essentially the result of replacing $X_{i}$ by $K_{i}$ and $A_{i}$ by $A_{i}^{*}$ in $\mathrm{AX}_{e}^{X, A, \forall}$, Theorem 4.2(b) makes precise the sense in which $K_{i}$ acts like $X_{i}$ with respect to $A_{i}^{*}$.

Dekel, Lipman, and Rustichini (1998) show that, in a precise sense, it is impossible to have an unawareness operator that satisfies certain properties in a standard state-space model. They consider semantic unawareness and knowledge operators, that is, functions from sets of states to sets of states. We do have nontrivial unawareness, so it is of interest to examine the properties they consider in our setting, to understand why their result does not apply.

They consider the following properties, where $\mathbf{K}$ and $\mathbf{U}$ denote the knowledge and unawareness operators, respectively (recall that these are functions from sets of states to sets of states), and $S$ is the set of states, and $E, F \subseteq S$ :

1. If $E \subseteq F$, then $\mathbf{K}(E) \subseteq \mathbf{K}(F)$

2. $\mathbf{K}(S)=S$.

3. $\mathbf{U}(E) \subseteq \mathbf{U}(\mathbf{U}(E))$

4. $\mathbf{U}(E) \subset \neg \mathbf{K}(E) \cap \neg \mathbf{K} \neg \mathbf{K}(E)$

5. $\mathbf{K}(\mathbf{U}(E))=\emptyset$. 
The first property says that knowledge is monotonic; if $E$ implies $F$ and you know $E$, then you should also know $F$. The second property essentially says that you know all tautologies. The third says that if you are unaware of something, you are unaware that you are unaware of it. The fourth says that if you are unaware of something, then you do not know it, nor do you know that you do not know it. The last property says that you cannot know that you are unaware of something.

When translating these properties to our framework, we represent unawareness as $\neg A$, the negation of awareness. With this interpretation, the third property becomes $\neg A \varphi \Rightarrow \neg A \neg A \varphi$, which is valid if awareness is generated by primitive propositions (although not in general). The validity of the remaining properties depends on whether we take $\mathbf{K}$ to represent implicit or explicit knowledge. Some of the properties are valid if we interpret $\mathbf{K}$ as implicit knowledge; others hold if we interpret it as explicit knowledge. But there is no one interpretation that makes all the properties valid. Property 1 (monotonicity) holds for implicit knowledge; if $\varphi \Rightarrow \psi$ is valid, so is $K \varphi \Rightarrow K \psi$. (Note that the subset relation corresponds to syntactic implication.) However, if $\varphi \Rightarrow \psi$ is valid, $X \varphi \Rightarrow X \psi$ may not be valid, since the agent may not be aware of $\psi$. Similarly, the second property is valid for implicit knowledge $(K \varphi$ is valid if $\varphi$ is valid), but is not valid for explicit knowledge (even if $\varphi$ is valid, $X \varphi$ may not be, because the agent may not be aware of $\varphi$ ). On the other hand, the last two properties are not valid for implicit knowledge, but are valid for explicit knowledge. Since there is no interpretation of knowledge that makes all the properties valid, the Dekel, Lipman, and Rustichini result does not apply in our setting. In a sense, it shows that the result depends on not distinguishing implicit and explicit knowledge.

\section{THE DEFINITION OF AWARENESS}

Just as in our framework, in the HMS and MR approach, a propositional language is associated with each world. However, HMS and MR define awareness of $\varphi$ as an abbreviation of $K_{i} \varphi \vee K_{i} \neg K_{i} \varphi$. In order to compare our approach to that of HMS and MR, we first compare the definitions of awareness. Let $A_{i}^{\prime} \varphi$ be an abbreviation for the formula $K_{i} \varphi \vee K_{i} \neg K_{i} \varphi$. Recall that $A_{i}^{*}(\varphi)$ is an abbreviation for the formula $K_{i}(\varphi \vee \neg \varphi)$. $A_{i}^{*} \varphi$ is syntactically quite different from $A_{i}^{\prime} \varphi$, and seems to be capturing different intuitions. Nevertheless, as the following result shows, in extended awareness 
structures that are Euclidean, $A_{i}^{*} \varphi$ is equivalent to $A_{i}^{\prime} \varphi$.

Proposition 5.1 If $M=\left(S, \mathcal{L}, \pi, \mathcal{K}_{1}, \ldots, \mathcal{K}_{n}, \mathcal{A}_{1}, \ldots, \mathcal{A}_{n}\right)$ is a Euclidean extended awareness structure, then for all $s \in S$ and all sentences $\varphi \in$ $\mathcal{L}_{n}^{\forall, K, X, A}(\Phi, \mathcal{X}),{ }^{10}$

$$
(M, s) \models A_{i}^{*} \varphi \Leftrightarrow A_{i}^{\prime} \varphi .
$$

Proof: Suppose that $(M, s) \models K_{i}(\varphi \vee \neg \varphi) \wedge \neg K_{i} \varphi$. It follows that $\Phi(\varphi) \subseteq$ $\mathcal{L}(s), \Phi(\varphi) \subseteq \mathcal{L}(t)$ for all $t$ such that $(s, t) \in \mathcal{K}_{i}$, and that there exists a world $t$ such that $(s, t) \in \mathcal{K}_{i}$ and $(M, t) \models \neg \varphi$. Let $u$ be an arbitrary world such that $(s, u) \in \mathcal{K}_{i}$. Since $\mathcal{K}_{i}$ is Euclidean, it follows that $(u, t) \in \mathcal{K}_{i}$. Thus, $(M, u) \models \neg K_{i} \varphi$, so $(M, s) \models K_{i} \neg K_{i} \varphi$. It follows that $(M, s) \models A_{i}^{\prime} \varphi$, as desired.

For the converse, suppose that $(M, s) \models A_{i}^{\prime} \varphi$. If either $(M, s) \models K_{i} \varphi$ or $(M, s) \models K_{i} \neg K_{i} \varphi$, then $\Phi(\varphi) \subseteq \mathcal{L}(s)$, and if $(s, t) \in \mathcal{K}_{i}$, we have that $\Phi(\varphi) \subseteq \mathcal{L}(t)$. Therefore, $(M, s) \models A_{i}^{*} \varphi$.

The assumption in Proposition 5.1 that the relation is Euclidean is necessary for $A_{i}^{*} \varphi$ and $A_{i}^{\prime} \varphi$ to coincide. To see this, consider an extended awareness structure with three states $s, t$, and $u$ such that $\mathcal{K}_{i}=\{(s, t),(s, u)\}$, $p \in \mathcal{L}(s) \cap \mathcal{L}(t) \cap \mathcal{L}(u)$ and $(M, t) \models \neg p$. Thus, $(M, u) \models K_{i} p$ and $(M, s) \models$ $K_{i}(p \vee \neg p) \wedge \neg K_{i} p \wedge \neg K_{i} \neg K_{i} p$, that is, $(M, s) \models A_{i}^{*} p \wedge \neg A_{i}^{\prime} p$.

In (Halpern and Rêgo, 2008), we showed that $\mathrm{AX}_{e}^{K, A^{*}} \cup\left\{\mathrm{T}, 4,5^{*}\right\}$ provides a sound and complete axiomatization of the structures used by HMS where the possibility relations are Euclidean, transitive, and reflexive, with one difference: $A_{i}^{\prime}$ is used for awareness instead of $A_{i}^{*}$. However, by Proposition 5.1, in $\mathcal{N}_{n}^{e}, A_{i}^{*}$ and $A_{i}^{\prime}$ are equivalent. Thus, for the class of structures of most interest, we are able to get all the properties of the HMS approach; moreover, we can accommodate reasoning about knowledge of unawareness. It is not clear how to capture knowledge of unawareness directly in the HMS approach.

It remains to consider the relationship between $A_{i}$ and $A_{i}^{*}$. Let $\mathcal{A}_{i}^{*}(s)$ be the set of sentences that can be expressed in the language at all the worlds considered possible by agent $i$ in world $s$; that is, $\varphi \in \mathcal{A}_{i}^{*}(s)$ iff $(M, s) \models A_{i}^{*} \varphi$. Assuming that agents know what they are aware of, we have that if $(s, t) \in \mathcal{K}_{i}$, then $\mathcal{A}_{i}(s)=\mathcal{A}_{i}(t)$. Thus, it follows that $\mathcal{A}_{i}(s) \subseteq \mathcal{A}_{i}^{*}(s)$.

\footnotetext{
${ }^{10}$ Dekel, Lipman, and Rustichini (1998) defined a property called weak necessitation. This property states that whenever an agent is aware of a formula, then he must know any tautology involving that formula. Proposition 5.1 implies that, for HMS and MR's definition of awareness, weak necessitation is valid in extended awareness structures. It is not hard to check that weak necessitation is also valid using our syntactic definition of awareness.
} 
For if $\varphi \in \mathcal{A}_{i}(s)$, then $\Phi(\varphi) \subseteq \mathcal{L}(t)$ for all $t$ such that $(s, t) \in \mathcal{K}_{i}$, so $(M, s) \models A_{i}^{*}(\varphi)$.

As discussed in Section 4, the opposite inclusion does not hold in general. But we can get it by assuming the following natural connection between an agent's awareness function and the language in the worlds that he considers possible:

- LA: If $p \in \mathcal{L}(s)-\mathcal{A}_{i}(s)$, then $p \notin \mathcal{L}(t)$ for some $t$ such that $(s, t) \in \mathcal{K}_{i}$.

LA essentially says that if an agent is unaware of $p$, then he should not implicitly know that he is unaware of $p .{ }^{11}$ Thus, he should not use the same label (i.e., $p$ ) in all worlds for a proposition that he is unaware of. Put another way, if the agent is unaware of $p$, he should consider it possible that $p$ does not exist at all. We find this condition quite natural. While we do not require it in general, it is just what is needed to get the equivalence of $\mathcal{A}_{i}$ and $\mathcal{A}_{i}^{*}$. More precisely, it is immediate that in models that satisfy LA (and agpp $), \mathcal{A}_{i}(s) \supseteq \mathcal{A}_{i}^{*}(s)$ for all agents $i$ and worlds $s$. Thus, under minimal assumptions, $\mathcal{A}_{i}^{*}(s)=\mathcal{A}_{i}(s)$. Moreover, under assumption LA (and agpp), $K_{i} \varphi$ and $X_{i} \varphi$ are equivalent.

Proposition 5.2 If $M$ is an extended awareness structure satisfying LA and agpp, then

(a) $(M, s) \models A_{i} \varphi \Leftrightarrow A_{i}^{*} \varphi$ and

(b) $(M, s) \models K_{i} \varphi \Leftrightarrow X_{i} \varphi$.

Proof: For part (a), clearly $(M, s) \models A_{i} \varphi$ iff $\varphi \in \mathcal{A}_{i}(s)$, and $(M, s) \models$ $A_{i}^{*} \varphi$ iff $\varphi \in \mathcal{A}_{i}^{*}(s)$. Since it follows from $\mathbf{L} \mathbf{A}$ and agpp that $\mathcal{A}_{i}(s)=\mathcal{A}_{i}^{*}(s)$, part (a) follows.

For part (b), it is immediate that $(M, s) \models X_{i} \varphi$ implies $(M, s) \models K_{i} \varphi$. Now, consider that $(M, s) \models K_{i} \varphi$. Thus, for every $p$ in $\varphi$ and $t$ such that $(s, t) \in \mathcal{K}_{i}$, we have $p \in \mathcal{L}(t)$, for otherwise, $\varphi$ would not be true at $t$. Since $(M, s) \models K_{i} \varphi$, it follows that for every $p$ in $\varphi$ we have $p \in \mathcal{L}(s)$. Therefore, LA implies that for every $p$ in $\varphi, p \in \mathcal{A}_{i}(s)$. By agpp, we get $(M, s) \models A_{i} \varphi$ as desired.

The bottom line here is that under the standard assumptions in the economics literature, together with the minimal assumption LA, all the notions of awareness coincide. We do not need to consider a syntactic notion

\footnotetext{
${ }^{11}$ Condition LA further restricts to formulas $p$ that are in $\mathcal{L}(s)$. This weaker condition suffices to prove our results.
} 
of awareness at all. However, as pointed out by FH, there are other notions of awareness that may be relevant; in particular, a more computational notion of awareness is of interest. For such a notion, an axiom such as AGPP does not seem appropriate. We leave the problem of finding axioms that characterize a more computational notion of awareness in this framework to future work.

Part (a) of the following proposition relates the HMS and MR definition of awareness, that is, the operator that we have called $A_{i}^{\prime}$, to $A_{i}$ and $X_{i}$. Specifically, it shows that $X_{i} \varphi \vee X_{i} \neg X_{i} \varphi$ is equivalent to $A_{i} \varphi \wedge A_{i}^{\prime} \varphi$; moreover, in Euclidean structures, $X_{i} \varphi \vee X_{i} \neg X_{i} \varphi$ is equivalent to $A_{i} \varphi$. Part (b) shows that, analogously, $X_{i}(\varphi \vee \neg \varphi)$ is equivalent to $A_{i} \varphi$. Combining both results, we get that $X_{i}(\varphi \vee \neg \varphi)$ is equivalent to $X_{i} \varphi \vee X_{i} \neg X_{i} \varphi$ in Euclidean structures.

Proposition 5.3 Let $M$ be an extended awareness structure satisfying agpp and $k a$.

(a) $(M, s) \models X_{i} \varphi \vee X_{i} \neg X_{i} \varphi$ iff $(M, s) \models A_{i} \varphi \wedge\left(K_{i} \varphi \vee K_{i} \neg K_{i} \varphi\right)$. Moreover, if $M$ is Euclidean, then $(M, s) \models X_{i} \varphi \vee X_{i} \neg X_{i} \varphi$ iff $(M, s) \models A_{i} \varphi$.

(b) $(M, s) \models X_{i}(\varphi \vee \neg \varphi)$ iff $(M, s) \models A_{i} \varphi$.

\section{DISCUSSION}

We have introduced a semantic model where we have both lack of awareness and different languages at each world. By allowing different languages at each world, we can capture natural states of knowledge of lack of awareness that cannot be captured using earlier frameworks. Moreover, it allows us to unify various approaches to unawareness. But doing this requires us to examine more carefully the connection between awareness and language.

Recall that we think of propositions $p \in \mathcal{L}(t)-\mathcal{A}_{i}(s)$ as just being labels for concepts that the agent is unaware of, but understands might be "out there" and relevant. For example, in the oncology example described in the introduction, the agent might be unaware of various cancer treatments. It is important that we allow there to be more than one primitive proposition in $\mathcal{L}(s)-\mathcal{A}_{i}(s)$. A world where an agent is unaware of two primitive propositions is different from a world where an agent is unaware of only one primitive proposition. For example, to express the fact that, in world $s, i$ is unaware of two cancer treatments, we could have two primitive propositions in $\mathcal{L}(s)-\mathcal{A}_{i}(s)$. 
The propositions in $\mathcal{L}(s)-\mathcal{A}_{i}(s)$ take on added significance if there are several agents in the picture. For example, if agent 1 considers it possible that (1) there is a formula that he is unaware that agent 2 is aware of and (2) there is a formula that both he and agent 2 are unaware of that agent 3 is aware of, agent 1 needs to consider possible a world $t$ with at least two primitive propositions in $\mathcal{L}(t)-\mathcal{A}_{1}(s)$. Needless to say, reasoning about such lack of awareness might be critical in a decision-theoretic context. Although it is unlikely that the agent would know that there are exactly three primitive propositions that he is unaware of, he might well consider it possible that there are between three and ten relevant concepts that he is unaware of. These examples suggest that the line between awareness and unawareness is not so sharp. Facts regarding unawareness may still impact an agent's decisions. We return to this issue below.

The fact that the primitive propositions that an agent is not aware of are simply labels means that switching the labels does not affect what the agent knows or believes. More precisely, given a model $M=\left(S, \mathcal{L}, \mathcal{K}_{1}, \ldots, \mathcal{K}_{n}, \mathcal{A}_{1}, \ldots, \mathcal{A}_{n}, \pi\right)$, let $M^{\prime}$ be identical to $M$ except that the roles of the primitive propositions $p$ and $p^{\prime}$ are interchanged. More formally, $M^{\prime}=\left(S, \mathcal{L}^{\prime}, \mathcal{K}_{1}, \ldots, \mathcal{K}_{n}, \mathcal{A}_{1}^{\prime}, \ldots, \mathcal{A}_{n}^{\prime}, \pi^{\prime}\right)$, where, for all worlds $s \in S$, we have

- $\mathcal{L}(s)-\left\{p, p^{\prime}\right\}=\mathcal{L}^{\prime}(s)-\left\{p, p^{\prime}\right\}$

- $p \in \mathcal{L}^{\prime}(s)$ iff $p^{\prime} \in \mathcal{L}(s)$, and $p^{\prime} \in \mathcal{L}^{\prime}(s)$ iff $p \in \mathcal{L}(s)$;

- $\pi(s, q)=\pi^{\prime}(s, q)$ for all $q \in \mathcal{L}(s)-\left\{p, p^{\prime}\right\}$;

- if $p \in \mathcal{L}(s)$, then $\pi(s, p)=\pi^{\prime}\left(s, p^{\prime}\right)$, and if $p^{\prime} \in \mathcal{L}(s)$, then $\pi\left(s, p^{\prime}\right)=$ $\pi^{\prime}(s, p)$;

- if $\varphi$ is a formula that mentions neither $p$ nor $p^{\prime}$, then $\varphi \in \mathcal{A}_{i}(s)$ iff $\varphi \in \mathcal{A}_{i}^{\prime}(s)$

- for any formula $\varphi$ that mentions either $p$ or $p^{\prime}, \varphi \in \mathcal{A}_{i}(s)$ iff $\varphi[p \leftrightarrow$ $\left.p^{\prime}\right] \in \mathcal{A}_{i}^{\prime}(s)$, where $\varphi\left[p \leftrightarrow p^{\prime}\right]$ is the result of replacing all occurrences of $p$ in $\varphi$ by $p^{\prime}$ and all occurrences of $p^{\prime}$ by $p$.

It is easy to see that for all worlds $s,(M, s) \models \varphi$ iff $\left(M^{\prime}, s\right) \models \varphi\left[p \leftrightarrow p^{\prime}\right]$. In particular, this means that if neither $p$ nor $p^{\prime}$ is in $\mathcal{L}(s)$, then for all formulas, $(M, s) \models \varphi$ iff $\left(M^{\prime}, s\right) \models \varphi$. Thus, switching labels of propositions that are not in $\mathcal{L}(s)$ has no impact on what is true at $s$.

We remark that the use of labels here is similar in spirit to our use of virtual moves in (Halpern and Rêgo, 2013) to model moves that a player is aware that he is unaware of. 
Note that we allow agents to have some partial information about formulas that they are unaware of. We certainly want to allow agent 1 to know that there is a formula that agent 2 is aware of that he (agent 1 ) is unaware of; indeed, capturing a situation like this was one of our primary motivations for introducing knowledge of lack of awareness. But we also want to allow agent 1 to know that agent 2 is not only aware of the formula, but knows that it is true; that is, we want $X_{1}\left(\exists x\left(\neg A_{1}(x) \wedge X_{2}(x)\right)\right)$ to be consistent (and, in fact, this formula is consistent in the model presented in this paper). There may come a point when an agent has so much partial information about a formula he is unaware of that, although he cannot talk about it explicitly in his language, he can describe it sufficiently well to communicate about it. When this happens in natural language, people will come up with a name for a concept and add it to their language. Again, this emphasizes the point that the line between awareness and lack of awareness is not so sharp. We have not addressed the dynamics of language change here, but we believe that this is a topic that deserves further research.

Like standard decision theory, where an agent's choice does not change if we relabel the events and acts, in our model, switching labels of propositions that are not in $\mathcal{L}(s)$ has no impact on what is true at $s$. However, changing the truth value of a primitive proposition that an agent is not aware at $s$ may have some impact on what the agent explicitly knows at $s$. For example, consider a model $M$ such that $\Phi=\{q\}, S=\{s, t\}, \pi(s, q)=$ true, $\pi(t, q)=$ false, $q \notin \mathcal{A}_{1}(s)=\mathcal{A}_{1}(t)$, and $\mathcal{K}_{1}=\{(s, s),(s, t),(t, s),(t, t)\}$. In this model, we have that $\varphi=X_{1}\left(\exists x\left(\neg A_{1} x \wedge \neg K_{1} x \wedge \neg K_{1} \neg x\right)\right)$ is true at $s$, since $\neg A_{1} q \wedge \neg K_{1} q \wedge \neg K_{1} \neg q$ holds at both worlds $s$ and $t$. However, if we were to change the truth value of $q$ from false to true in $t$, then $\varphi$ would no longer be true at $s$; this change would make the worlds $s$ and $t$ identical, so the same formulas would be true at both. We can get a similar result without using the $K$ operator by adding another agent to the model, taking $\mathcal{K}_{2}=\mathcal{K}_{1}$, setting $\mathcal{A}_{2}(s)=\mathcal{A}_{2}(t)=\{q\}$, and replacing $K_{1}$ by $X_{2}$.

More generally, it seems natural to consider the impact of awareness on decision making. First steps in this direction were taken by Schipper (2010). He worked in the awareness structures introduced by Heifetz, Meier, and Schipper (2006; 2008), and thus did not deal with awareness of unawareness. While an agent cannot make decisions based on facts that he is unaware of, it is clear that awareness of unawareness can have a significant impact in decision making. In terms of our earlier discussion, we are saying that the different levels of unawareness can have an impact on decision making. We leave the problem of finding an appropriate decision model and clarifying the impact to future work. 


\section{A PROOFS}

We first prove Theorem 4.1. As we said in the main text, proving soundness turns out to be nontrivial, so we begin by showing that MP, Barcan ${ }_{X}^{*}$, and $\mathrm{Gen}_{\forall}$ are sound. (Soundness of the remaining axioms is straightforward.) For MP, we need some preliminary lemmas.

Lemma A.1 If $\varphi$ is a sentence in $\mathcal{L}_{n}^{\forall, K, X, A}(\Phi, \mathcal{X})$ that does not mention $q$ and is satisfiable in $\mathcal{N}_{n}(\Phi, \mathcal{X})$, then it is satisfiable in an extended awareness structure $M=\left(S, \mathcal{L}(s), \pi, \mathcal{K}_{1}, \ldots, \mathcal{K}_{n}, \mathcal{A}_{1}, \ldots, \mathcal{A}_{n}\right) \in \mathcal{N}_{n}(\Phi, \mathcal{X})$ such that $q \notin \mathcal{L}(s)$ for every $s \in S$.

Proof: Let $\tau: \Phi \rightarrow \Phi$ be a 1-1 function. For a sentence $\psi$, let $\tau(\psi)$ be the result of replacing every primitive proposition $q$ in $\psi$ by $\tau(q)$. Given an extended awareness structure $M^{\prime}=\left(S, \mathcal{L}(s), \pi, \mathcal{K}_{1}, \ldots, \mathcal{K}_{n}, \mathcal{A}_{1}, \ldots, \mathcal{A}_{n}\right)$, let $M^{\tau}=\left(S, \mathcal{L}^{\tau}(s), \pi^{\tau}, \mathcal{K}_{1}, \ldots, \mathcal{K}_{n}, \mathcal{A}_{1}^{\tau}, \ldots, \mathcal{A}_{n}^{\tau}\right)$ be the extended awareness structure that results from "translating" $M$ by $\tau$; formally: $\mathcal{L}^{\tau}(s)=\{\tau(p)$ : $p \in \mathcal{L}(s)\}, \pi^{\tau}(s, \tau(p))=\pi(s, p)$, and $\mathcal{A}_{i}^{\tau}(s)=\left\{\tau(\psi): \psi \in \mathcal{A}_{i}(s)\right\}$. We now prove that $(M, s) \models \psi$ iff $\left(M^{\tau}, s\right) \models \tau(\psi)$ by induction in the structure of $\psi$. All the cases are straightforward and left to the reader except the case $\psi$ has the form $\forall x \psi^{\prime}$. In this case, we have that $(M, s) \models \psi$ iff $(M, s) \models \psi^{\prime}[x / \beta]$ for all $\beta \in \mathcal{L}_{n}^{K, X, A}(\mathcal{L}(s))$. By the induction hypothesis, $(M, s) \models \psi^{\prime}[x / \beta]$ for all $\beta \in \mathcal{L}_{n}^{K, X, A}(\mathcal{L}(s))$ iff $\left(M^{\tau}, s\right) \models \tau\left(\psi^{\prime}[x / \beta]\right)$ for all $\beta \in \mathcal{L}_{n}^{K, X, A}(\mathcal{L}(s))$. Since $\tau\left(\psi^{\prime}[x / \beta]\right)=\tau\left(\psi^{\prime}\right)[x / \tau(\beta)]$ and, by construction of $\mathcal{L}^{\tau}$, for all $\gamma \in \mathcal{L}_{n}^{K, X, A}\left(\mathcal{L}^{\tau}(s)\right)$ there exists $\beta \in \mathcal{L}_{n}^{K, X, A}(\mathcal{L}(s))$ such that $\gamma=\tau(\beta)$, it follows that $\left(M^{\tau}, s\right) \models \tau\left(\psi^{\prime}[x / \beta]\right)$ for all $\beta \in \mathcal{L}_{n}^{K, X, A}(\mathcal{L}(s))$ iff $\left(M^{\tau}, s\right) \models \tau\left(\psi^{\prime}\right)[x / \gamma]$ for all $\gamma \in \mathcal{L}_{n}^{K, X, A}\left(\mathcal{L}^{\tau}(s)\right)$. The latter statement is true iff $\left(M^{\tau}, s\right) \models \tau(\psi)$.

To complete the proof of the lemma, suppose that $\varphi$ is a sentence that does not mention $q$ and that $(M, s) \models \varphi$. Let $\tau$ be a 1-1 function such that $\tau(p)=p$ for every $p$ that occurs in $\varphi$ and such that there exists no $r \in \Phi$ such that $\tau(r)=q$. (Here we are using the fact that $\Phi$ is an infinite set.) Note that $\varphi=\tau(\varphi)$. Thus, the claim implies that $\left(M^{\prime}, s\right) \models \varphi$ and by construction $q \notin \mathcal{L}^{\prime}(s)$ for every $s \in S$.

Substitution is a standard property of most propositional logics. It says that if $\varphi$ is valid, then so is $\varphi[q / \psi]$. Substitution in full generality is not valid in our framework, because of the semantics of quantification. For example, although $\forall x \neg A_{i} x \Rightarrow \neg A_{i} q$ is valid, $\forall x \neg A_{i} x \Rightarrow \neg A_{i}\left(\forall x A_{i} x\right)$ is not. As we now show, if we restrict to quantifier-free substitutions, we preserve validity. But this result depends on the fact that $\Phi$ is infinite. 
For example, if $\Phi=\{p, q\}$, then $\varphi=A_{i} p \wedge A_{i} q \Rightarrow \forall x A_{i} x$ is valid, but $\varphi[q / p]=A_{i} p \wedge A_{i} p \Rightarrow \forall x A_{i} x$ is not valid. We first prove that a slightly weaker version of Substitution holds (in which $q$ cannot appear in $\psi$ ), and then prove Substitution.

Proposition A.1 (Weak Substitution) If $\varphi$ is a sentence valid in $\mathcal{N}_{n}(\Phi, \mathcal{X})$, $q$ is a primitive proposition, and $\psi$ is an arbitrary quantifier-free sentence that does not mention $q$, then $\varphi[q / \psi]$ is valid in $\mathcal{N}_{n}(\Phi, \mathcal{X})$.

Proof: Suppose, by way of contradiction, that $\varphi[q / \psi]$ is not valid. Then $\neg \varphi[q / \psi]$ is satisfiable. By Lemma A.1, there exists an extended awareness structure $M=\left(S, \mathcal{L}(s), \pi, \mathcal{K}_{1}, \ldots, \mathcal{K}_{n}, \mathcal{A}_{1}, \ldots, \mathcal{A}_{n}\right)$ and a world $s^{*} \in S$ such that $\left(M, s^{*}\right) \models \neg \varphi[q / \psi]$ and $q \notin \mathcal{L}(s)$ for every $s \in S$. Let $M^{\prime}$ extends $M$ by defining $q$ as $\psi$; more precisely, $M^{\prime}=\left(S, \mathcal{L}^{\prime}, \pi^{\prime}, \mathcal{K}_{1}, \ldots, \mathcal{K}_{n}, \mathcal{A}_{1}^{\prime}, \ldots, \mathcal{A}_{n}^{\prime}\right)$, where

- $\mathcal{L}^{\prime}(s)=\mathcal{L}(s) \cup\{q\}$ if $\psi \in \mathcal{L}_{n}^{K, X, A}(\mathcal{L}(s))$, and $\mathcal{L}^{\prime}(s)=\mathcal{L}(s)$ otherwise;

- $\pi^{\prime}(s, p)=\pi(s, p)$ for every $p \in \mathcal{L}(s)$ and if $q \in \mathcal{L}^{\prime}(s)$, then $\pi^{\prime}(s, q)=$ true iff $(M, s) \models \psi$;

- $\mathcal{A}_{i}^{\prime}(s)=\mathcal{A}_{i}(s)$ if $\psi \notin \mathcal{A}_{i}(s)$, and $\mathcal{A}_{i}^{\prime}(s)$ is the smallest set generated by primitive propositions that includes $\mathcal{A}_{i}(s) \cup\{q\}$ otherwise.

Intuitively, we are just extending $M$ by defining $q$ so that it agrees with $\psi$ everywhere. We claim that for every sentence $\sigma$, if $\psi \in \mathcal{L}_{n}^{K, X, A}(\mathcal{L}(s))$, then the following are equivalent:

(a) $\left(M^{\prime}, s\right) \models \sigma$

(b) $\left(M^{\prime}, s\right) \models \sigma[q / \psi]$

(c) $(M, s) \models \sigma[q / \psi]$.

We first observe that if $\sigma^{\prime}$ is a quantifier-free sentence that does not mention $q$, then for all worlds $s \in S$, we have that $(M, s) \models \sigma$ iff $\left(M^{\prime}, s\right) \models \sigma^{\prime}$. (The formal proof is by a straightforward induction on $\sigma^{\prime}$.)

We now prove the claim by induction in the structure of $\sigma$. For the base case, note that if $\sigma$ is the primitive proposition $q$, then the equivalence between (b) and (c) follows from the observation above. All cases are straightforward except the case where $\sigma$ has the form $\forall x \sigma^{\prime}$. To see that (a) implies (b), suppose that $\left(M^{\prime}, s\right) \models \forall x \sigma^{\prime}$. Then $\left(M^{\prime}, s\right) \models \sigma^{\prime}[x / \beta]$ for all $\beta \in \mathcal{L}_{n}^{K, X, A}\left(\mathcal{L}^{\prime}(s)\right)$. By the induction hypothesis, $\left(M^{\prime}, s\right) \models\left(\sigma^{\prime}[x / \beta]\right)[q / \psi]$. 
Note that $\sigma^{\prime}[x / \beta][q / \sigma]=\left(\left(\sigma^{\prime}[q / \psi]\right)[x / \beta]\right)[q / \psi]$. Thus, applying the induction hypothesis again, it follows that $\left(M^{\prime}, s\right) \models\left(\sigma^{\prime}[q / \psi]\right)[x / \beta]$ for all $\beta \in \mathcal{L}_{n}^{K, X, A}\left(\mathcal{L}^{\prime}(s)\right)$. Therefore, $\left(M^{\prime}, s\right) \models \forall x \sigma^{\prime}[q / \psi]$. This shows that (a) implies (b).

To see that (b) implies (c), suppose that $\left(M^{\prime}, s\right) \models \forall x \sigma^{\prime}[q / \psi]$. Thus, $\left(M^{\prime}, s\right) \models\left(\sigma^{\prime}[q / \psi]\right)[x / \beta]$ for all $\beta \in \mathcal{L}_{n}^{K, X, A}\left(\mathcal{L}^{\prime}(s)\right)$. Since $\mathcal{L}_{n}^{K, X, A}(\mathcal{L}(s)) \subseteq$ $\mathcal{L}_{n}^{K, X, A}\left(\mathcal{L}^{\prime}(s)\right)$, by the induction hypothesis, it follows that $(M, s) \models\left(\sigma^{\prime}[q / \psi]\right)[x / \beta]$ for all $\beta \in \mathcal{L}_{n}^{K, X, A}(\mathcal{L}(s))$. Thus, $(M, s) \models \forall x \sigma^{\prime}[q / \psi]$.

Finally, to see that (c) implies (a), suppose that $(M, s) \models \forall x \sigma^{\prime}[q / \psi]$. We want to show that $\left(M^{\prime}, s\right) \models \forall x \sigma^{\prime}$, or equivalently, that $\left(M^{\prime}, s\right) \models$ $\sigma^{\prime}[x / \beta]$ for all $\beta \in \mathcal{L}_{n}^{K, X, A}\left(\mathcal{L}\left(s^{\prime}\right)\right)$. Choose $\beta \in \mathcal{L}_{n}^{K, X, A}\left(\mathcal{L}\left(s^{\prime}\right)\right)$. So choose $\beta \in \mathcal{L}_{n}^{K, X, A}\left(\mathcal{L}\left(s^{\prime}\right)\right)$. By the induction hypothesis, $\left(M^{\prime}, s\right) \models \sigma^{\prime}[x / \beta]$ iff $\left(M^{\prime}, s\right) \models\left(\sigma^{\prime}[x / \beta]\right)[q / \psi]$ iff $(M, s) \models\left(\sigma^{\prime}[x / \beta]\right)[q / \psi]$. Since $\left(\sigma^{\prime}[x / \beta]\right)[q / \psi]=$ $\sigma^{\prime}[q / \sigma](x / \beta[q / \sigma])$, and $(M, s) \models \sigma^{\prime}[q / \sigma](x / \beta[q / \sigma])$ since $(M, s) \models \forall x \sigma^{\prime}[q / \sigma]$, by assumption, the desired result follows.

Since, by assumption, $\left(M, s^{*}\right) \models \neg \varphi[q / \psi]$, it follows from the claim above that $\left(M^{\prime}, s^{*}\right) \models \neg \varphi$, a contradiction.

Corollary A.1 (Substitution) If $\varphi$ is a sentence valid in $\mathcal{N}_{n}(\Phi, \mathcal{X}), q$ is a primitive proposition, and $\psi$ is an arbitrary quantifier-free sentence, then $\varphi[q / \psi]$ is valid in $\mathcal{N}_{n}(\Phi, \mathcal{X})$.

Proof: Choose a primitive proposition $r$ that does not appear in $\psi$ or $\varphi$. By Weak Substitution (Proposition A.1), $\varphi^{\prime}=\varphi[q / r]$ is valid. Applying Weak Substitution again, $\varphi^{\prime}[r / \psi]=\varphi[q / \psi]$ is valid.

We are finally ready to prove the soundness of MP.

Corollary A.2 If $\varphi \Rightarrow \psi$ and $\varphi$ are both valid in an awareness structure $M$, then so is $\varphi$.

Proof: Suppose, by way of contradiction, then $\varphi \Rightarrow \psi$ and $\varphi$ are valid in $M$, and, for some world $s$ in $M$, we have that $(M, s) \models \neg \varphi$. It must be the case that $\psi \notin \mathcal{L}_{n}^{\forall, K, X, A}(\mathcal{L}(s), \mathcal{X})$, while $\varphi \in \mathcal{L}_{n}^{\forall, K, X, A}(\mathcal{L}(s), \mathcal{X})$. Let $q_{1}, \ldots, q_{k}$ be the primitive propositions that are mentioned in $\psi$ but are not in $\mathcal{L}(s)$. Note that none of $q_{1}, \ldots, q_{k}$ can appear in $\varphi$. Since, by assumption, $\mathcal{L}(s)$ is nonempty, let $p \in \mathcal{L}(s)$, and let $\psi^{\prime}=\psi\left[q_{1} / p, \ldots, q_{k} / p\right]$. By Weak Substitution, $\psi^{\prime}$ and $\psi^{\prime} \Rightarrow \varphi$ are valid. But $\psi^{\prime}$ and $\varphi$ are in $\mathcal{L}_{n}^{\forall, K, X, A}(\mathcal{L}(s), \mathcal{X})$. Thus, we must have $(M, s) \models \psi^{\prime}$ and $(M, s) \models \psi^{\prime} \Rightarrow \varphi$, so $(M, s) \models \varphi$, a contradiction.

The following two results prove the soundness of $\mathrm{Gen}_{\forall}$ and $\operatorname{Barcan}_{X}^{*}$. 
Proposition A.2 $\left(\mathbf{G e n}_{\forall}\right)$ If $\varphi$ is a valid sentence in $\mathcal{N}_{n}(\Phi, \mathcal{X})$ and $q$ is an arbitrary primitive proposition, then $\forall x \varphi[q / x]$ is valid in $\mathcal{N}_{n}(\Phi, \mathcal{X})$.

Proof: Suppose not. Then there exists an extended awareness structure in $M \in \mathcal{N}_{n}(\Phi, \mathcal{X})$ and a world $s$ such that $(M, s) \models \neg \forall x \varphi[q / x]$. Thus, there exists a formula $\psi \in \mathcal{L}_{n}^{K, X, A}(\mathcal{L}(s))$ such that $(M, s) \models \neg(\varphi[q / x])[x / \psi]$. Thus, $\varphi[q / \psi]$ is not valid. By Substitution, it follows that $\varphi$ is not valid either, a contradiction.

Proposition A.3 $\left(\operatorname{Barcan}_{X}^{*}\right)\left(A_{i}(\forall x \varphi) \wedge \forall x\left(A_{i} x \Rightarrow X_{i} \varphi\right)\right) \Rightarrow X_{i}\left(\forall x A_{i} x \Rightarrow\right.$ $\forall x \varphi)$ is valid in $\mathcal{N}_{n}(\Phi, \mathcal{X})$.

Proof: Suppose that $(M, s) \models\left(A_{i}(\forall x \varphi) \wedge \forall x\left(A_{i} x \Rightarrow X_{i} \varphi\right)\right)$. Since awareness is generated by primitive propositions, $(M, s) \models A_{i}\left(\forall x A_{i} x \Rightarrow \forall x \varphi\right)$. Suppose, by way of contradiction, that $(M, s) \models \neg X_{i}\left(\forall x A_{i} x \Rightarrow \forall x \varphi\right)$. Then there must exist some world $t$ such that $(s, t) \in \mathcal{K}_{i}$ and $(M, t) \models \neg\left(\forall x A_{i} x \Rightarrow\right.$ $\forall x \varphi)$. Thus, $(M, t) \models \forall x A_{i} x$ and $(M, t) \models \neg \forall x \varphi$. Since $(M, t) \models \neg \forall x \varphi$, it follows that there exists $\psi \in \mathcal{L}_{n}^{X, A}(\mathcal{L}(t))$ such that $(M, t) \models \neg \varphi[x / \psi]$. Since $(M, t) \models \forall x A_{i} x$, we must have $(M, t) \models A_{i} \psi$. Since $\mathcal{A}_{i}(s)=\mathcal{A}_{i}(t)$, we also have $(M, s) \models A_{i} \psi$. Since $(M, s) \models \forall x\left(A_{i} x \Rightarrow X_{i} \varphi\right)$, it follows that $(M, s) \models X_{i} \varphi[x / \psi]$. Thus, $(M, t) \models \varphi[x / \psi]$, a contradiction.

With these results in hand, we can now prove Theorem 4.1. We repeat the theorem here for the convenience of the reader.

Theorem 4.1: If $\mathcal{C}_{X}$ is a (possibly empty) subset of $\left\{\mathrm{T}_{\mathrm{X}}, 4_{\mathrm{X}}, 5_{\mathrm{X}}\right\}$ and $C$ is the corresponding subset of $\{r, t, e\}$, then $\mathrm{AX}_{e}^{X, A, \forall} \cup \mathcal{C}_{X}$ is a sound and complete axiomatization of the language $\mathcal{L}_{n}^{\forall, X, A}(\Phi, \mathcal{X})$ with respect to $\mathcal{N}_{n}^{C}(\Phi, \mathcal{X})$

Proof: Corollary A.2 and Propositions A.2 and A.3 show the soundness of MP, Gen $\forall$, and $\operatorname{Barcan}_{X}^{*}$, respectively. The proof of soundness for the other axioms and rules is standard and left to the reader. The soundness of $\mathrm{AX}_{e}^{X, A, \forall} \cup \mathcal{C}_{X}$ follows easily.

We now consider completeness. As we said in the main text, the proof is quite similar in spirit to that of Theorem 2.1 given in HR. We focus here on the differences. We give the remainder of the proof only for the case $\mathcal{C}_{X}=\emptyset$; the other cases follow using standard techniques (see, for example, (Fagin et al., 1995; Hughes and Cresswell, 1996)).

As usual, the idea of the completeness proof is to construct a canonical model $M^{c}$ where the worlds are maximal consistent sets of sentences. It is then shown that if $s_{V}$ is the world corresponding to the maximal consistent set $V$, then $\left(M^{c}, s_{V}\right) \models \varphi$ iff $\varphi \in V$. As observed in HR, this will 
not quite work in the presence of quantification, since there may be a maximal consistent set $V$ of sentences such that $\neg \forall x \varphi \in V$, but $\varphi[x / \psi]$ for all $\psi \in \mathcal{L}_{n}^{K, X, A}(\Phi)$. That is, there is no witness to the falsity of $\forall x \varphi$ in $V$. This problem was dealt with in HR by restricting to maximal consistent sets $V$ that are acceptable in the sense that if $\neg \forall x \varphi \in V$, then $\neg \varphi[x / q] \in V$ for infinitely many primitive propositions $q \in \Phi$. (Note that this notion of acceptability also requires $\Phi$ to be infinite.) Because here we have possibly different languages associated different worlds, we need to consider acceptability and maximality with respect to a language.

Definition A.1 $A$ set $\Gamma$ is acceptable with respect to $L \subseteq \Phi$ if $\varphi \in$ $\mathcal{L}_{n}^{\forall, X, A}(L, \mathcal{X})$ and $\Gamma \vdash \varphi[x / q]$ for all but finitely many primitive propositions $q \in L$, then $\Gamma \vdash \forall x \varphi$.

Definition A.2 If $A X$ is an axiom system, a set $\Gamma$ is maximal AX-consistent set of sentences with respect to $L \subseteq \Phi$ if $\Gamma$ is a set of sentences contained in $\mathcal{L}_{n}^{\forall, X, A}(L, \mathcal{X})$ and, for all sentences $\varphi \in \mathcal{L}_{n}^{\forall, X, A}(L, \mathcal{X})$, if $\Gamma \cup\{\varphi\}$ is $A X$ consistent, then $\varphi \in \Gamma$.

The following four lemmas are essentially Lemmas A.4, A.5, A.6, and A.7 in HR. Since the proofs are essentially identical, we do not repeat them here.

Lemma A.2 If $\Gamma$ is a finite set of sentences, then $\Gamma$ is acceptable with respect to every subset $L \subseteq \Phi$ that contains infinitely many primitive propositions.

Lemma A.3 If $\Gamma$ is acceptable with respect to $L$ and $\tau$ is a sentence in $\mathcal{L}_{n}^{\forall, X, A}(L, \mathcal{X})$, then $\Gamma \cup\{\tau\}$ is acceptable with respect to $L$.

Lemma A.4 If $\Gamma \subseteq \mathcal{L}_{n}^{\forall, X, A}(L, \mathcal{X})$ is an acceptable $\mathrm{AX}_{e}^{X, A, \forall}$-consistent set of sentences with respect to $L$, then $\Gamma$ can be extended to a set of sentences that is acceptable and maximal $\mathrm{AX}_{e}^{X, A, \forall}$-consistent with respect to $L$.

Let $\Gamma / X_{i}=\left\{\varphi: X_{i} \varphi \in \Gamma\right\}$.

Lemma A.5 If $\Gamma$ is a a set of sentences that is maximal $\mathrm{AX}_{e}^{X, A, \forall}$-consistent with respect to $L$ containing $\neg X_{i} \varphi$ and $A_{i} \varphi$, then $\Gamma / X_{i} \cup\{\neg \varphi\}$ is $\operatorname{AX}_{e}^{X, A, \forall}$ consistent. 
Lemma A.14 in HR shows that if $\Gamma$ is an acceptable maximal consistent set that contains $A_{i} \varphi$ and $\neg X_{i} \varphi$, then $\Gamma / X_{i} \cup\{\neg \varphi\}$ can be extended to an acceptable maximal consistent set $\Delta$. (Lemma A.8 in HR proves a similar result for the $K_{i}$ operator.) The following lemma proves an analogous result, but here we must work harder to take the language into account. That is, we have to define the language $L^{\prime}$ with respect to which $\Delta$ is maximal and acceptable. As usual, we say that $L$ is co-infinite if $\Phi-L$ is infinite.

Lemma A.6 If $\Gamma$ is an acceptable maximal $\mathrm{AX}_{e}^{X, A,{ }_{-}}$-consistent set of sentences with respect to $L$, where $L$ is infinite and co-infinite, $\neg X_{i} \varphi \in \Gamma$, and $A_{i} \varphi \in \Gamma$, then there exist an infinite and co-infinite set $L^{\prime} \subseteq \Phi$ and a set $\Delta$ of sentences that is acceptable, maximal $\mathrm{AX}_{e}^{X, A, \forall}$-consistent with respect to $L^{\prime}$ and contains $\Gamma / X_{i} \cup\{\neg \varphi\}$. Moreover, $A_{i} \psi \in \Delta$ iff $A_{i} \psi \in \Gamma$ for all formulas $\psi$.

Proof: By Lemma A.5, $\Gamma / X_{i} \cup\{\neg \varphi\}$ is $\mathrm{AX}_{e}^{X, A,{ }_{\text {-consistent. We define }}}$ a subset $L^{\prime} \subseteq \Phi$ and construct a set $\Delta$ of sentences that is acceptable and maximal $\mathrm{AX}_{e}^{X, A,{ }^{\prime}}$-consistent with respect to $L^{\prime}$ such that $\Delta$ contains $\Gamma / X_{i} \cup\{\neg \varphi\}$ and $A_{i} \varphi \in \Delta$ iff $A_{i} \varphi \in \Gamma$ for all formulas $\varphi$.

We consider two cases: (1) $\Gamma / X_{i} \cup\{\neg \varphi\} \vdash \forall x A_{i} x$; and (2) $\Gamma / X_{i} \cup\{\neg \varphi\} \forall$ $\forall x A_{i} x$.

If $\Gamma / X_{i} \cup\{\neg \varphi\} \vdash \forall x A_{i} x$, then define $L^{\prime}=\left\{q: A_{i} q \in \Gamma\right\}$. Note that since $\Gamma \vdash A_{i} \varphi$, it follows that every primitive proposition $q$ in $\varphi$ must be in $L^{\prime}$, as is every primitive proposition in a formula in $\Gamma / X_{i} . L^{\prime}$ must be infinite, for if it were finite, then we would have that $\Gamma \vdash A_{i} q$ for only finitely many primitive propositions in $L$. Since $\Gamma$ is a maximal $\mathrm{AX}_{e}^{X, A,{ }_{-}}$-consistent set, it must be the case that $\Gamma \vdash \neg A_{i} q$ for all but finitely many primitive propositions $q \in L$. Since $\Gamma$ is acceptable with respect to $L, \Gamma \vdash \forall x \neg A_{i} x$. Thus, axiom $\mathrm{FA}_{X}^{*}$ implies that $\forall x \neg A_{i} x \in \Gamma / X_{i}$, which is a contradiction, since by assumption $\Gamma / X_{i} \cup\{\neg \varphi\} \vdash \forall x A_{i} x$. Thus, $L^{\prime}$ must be infinite. Since $L^{\prime}$ is a subset of $L$, it is clearly co-infinite, since $L$ is.

We prove that $\Gamma / X_{i} \cup\{\neg \varphi\}$ is acceptable with respect to $L^{\prime}$ in this case. Suppose that $\psi \in \mathcal{L}_{n}^{\forall, X, A}\left(L^{\prime}, \mathcal{X}\right)$ and

$$
\Gamma / X_{i} \cup\{\neg \varphi\} \vdash \psi[x / q] \text { for all but finitely many } q \in L^{\prime} \text {. }
$$

We want to show that $\Gamma / X_{i} \cup\{\neg \varphi\} \vdash \forall x \psi$. It follows from (2) that $\Gamma / X_{i} \vdash \neg \varphi \Rightarrow \psi[x / q]$ for all but finitely many $q \in L^{\prime}$. Since every primitive proposition in $\psi$ is in $L^{\prime}=\left\{q: A_{i} q \in \Gamma\right\}$, and $A_{i} \varphi \in \Gamma$, it easily follows that $\Gamma \vdash X_{i}(\neg \varphi \Rightarrow \psi[x / q])$ for all but finitely many $q \in L^{\prime}$. Since 
$L^{\prime}=\left\{q: A_{i} q \in \Gamma\right\}$, it follows that $\Gamma \vdash A_{i} q \Rightarrow X_{i}(\neg \varphi \Rightarrow \psi[x / q])$ for all but finitely many $q \in L$. Since $\Gamma$ is acceptable with respect to $L$, we have that

$$
\Gamma \vdash \forall x\left(A_{i} x \Rightarrow X_{i}(\neg \varphi \Rightarrow \psi)\right) .
$$

Again using the fact that $\Gamma \vdash A_{i} q$ for all $q$ in $\psi$ and $\Gamma \vdash A_{i} \varphi$, from AGPP we have that

$$
\Gamma \vdash A_{i} \forall x(\neg \varphi \Rightarrow \psi) .
$$

From $\operatorname{Barcan}_{X}^{*},(3)$, and (4), it follows that $\Gamma \vdash X_{i}\left(\forall x A_{i} x \Rightarrow \forall x(\neg \varphi \Rightarrow \psi)\right)$. Thus, $\Gamma / X_{i} \vdash \forall x A_{i} x \Rightarrow \forall x(\neg \varphi \Rightarrow \psi)$. Since $\Gamma / X_{i} \cup\{\neg \varphi\} \vdash \forall x A_{i} x$, it follows that $\Gamma / X_{i} \cup\{\neg \varphi\} \vdash \forall x(\neg \varphi \Rightarrow \psi)$. Since $\varphi$ is a sentence, applying $K_{\forall}$ and $N_{\forall}$, it easily follows that $\Gamma / X_{i} \cup\{\neg \varphi\} \vdash \neg \varphi \Rightarrow \forall x \psi$. Thus, $\Gamma / X_{i} \cup\{\neg \varphi\} \vdash \forall x \psi$, as desired.

Therefore, $\Gamma / X_{i} \cup\{\neg \varphi\}$ is a set of sentences that is acceptable with respect to $L^{\prime}$ and $\mathrm{AX}_{e}^{X, A,{ }^{-}}$-consistent. Thus, by Lemma A.4, there exists a set of sentences $\Delta$ containing $\Gamma / X_{i} \cup\{\neg \varphi\}$ that is acceptable and maximal $\operatorname{AX}_{e}^{X, A,{ }^{\prime}}$-consistent with respect to $L^{\prime}$. Finally, we prove that $A_{i} \psi \in \Gamma$ iff $A_{i} \psi \in \Delta$. First, suppose that $A_{i} \psi \in \Gamma$. Then, XA implies that $X_{i} A_{i} \psi \in \Gamma$. Thus, $A_{i} \psi \in \Gamma / X_{i} \subseteq \Delta$. For the converse, suppose that $A_{i} \psi \in \Delta$. Since $\psi \in \mathcal{L}_{n}^{\forall, X, A}\left(L^{\prime}, \mathcal{X}\right)$, it must be the case that $\Gamma \vdash A_{i} q$ for every primitive proposition $q$ that appears in $\psi$; thus $\Gamma \vdash A_{i} \psi$.

If $\Gamma / X_{i} \cup\{\neg \varphi\} \forall \forall \forall x A_{i} x$, define $L^{\prime}=\left\{q: A_{i} q \in \Gamma\right\} \cup L^{\prime \prime}$, where $L^{\prime \prime}$ is an infinite and co-infinite set of primitive propositions not occurring in $\Gamma \cup\{\varphi\}$ (which exists, since, by assumption, $\Phi-L$ is infinite). It can be easily seen that $L^{\prime}$ is infinite and co-infinite. Since $\Gamma / X_{i} \cup\{\neg \varphi\}$ is $\operatorname{AX}_{e}^{X, A, \forall_{-}}$ consistent, $\Gamma / X_{i} \cup\{\neg \varphi\} \forall \forall \forall x A_{i} x$ implies that $\Gamma / X_{i} \cup\left\{\neg \varphi, \neg \forall x A_{i} x\right\}$ is $\mathrm{AX}_{e}^{X, A,{ }^{\prime} \text {-consistent. }}$

To see that $\Gamma / X_{i} \cup\{\neg \varphi\}$ is acceptable with respect to $L^{\prime}$, suppose that $\psi \in \mathcal{L}_{n}^{\forall, X, A}\left(L^{\prime}, \mathcal{X}\right)$ and $\Gamma / X_{i} \cup\{\neg \varphi\} \vdash \psi[x / q]$ for all but finitely many $q \in L^{\prime}$. There must be some $q \in L^{\prime}$ not mentioned in $\Gamma / X_{i}$ or $\varphi$ such that $\Gamma / X_{i} \cup\{\neg \varphi\} \vdash \psi[x / q]$. Since $\Gamma / X_{i} \cup\{\neg \varphi\} \vdash \psi[x / q]$, it follows that there exists a subset $\left\{\beta_{1} \ldots, \beta_{n}\right\} \subseteq \Gamma / X_{i} \cup\{\neg \varphi\}$ such that $\mathrm{AX}_{e}^{X, A, \forall} \vdash \beta \Rightarrow \psi[x / q]$, where $\beta=\beta_{1} \wedge \cdots \wedge \beta_{n}$. Since $q$ does not occur in $\beta$ or $\varphi$, by $\operatorname{Gen}_{\forall}$, we have $\operatorname{AX}_{e}^{X, A, \forall} \vdash \forall x(\beta \Rightarrow \psi)$. Since $\beta$ is a sentence, applying $K_{\forall}$ and $N_{\forall}$, it easily follows that $\mathrm{AX}_{e}^{X, A, \forall} \vdash \beta \Rightarrow \forall x \psi$, which implies that $\Gamma / X_{i} \cup\{\neg \varphi\} \vdash \forall x \psi$, as desired. Finally, since $\Gamma / X_{i} \cup\{\neg \varphi\}$ is acceptable with respect to $L^{\prime}$, Lemma A.3 implies that $\Gamma / X_{i} \cup\left\{\neg \varphi, \neg \forall x A_{i} x\right\}$ is acceptable with respect to $L^{\prime}$.

Let $\psi_{1}, \psi_{2}, \ldots$ be an enumeration of the set of sentences in $\mathcal{L}_{n}^{\forall, X, A}\left(L^{\prime}, \mathcal{X}\right)$ such that if $\psi_{k}$ is of the form $\neg \forall x \varphi$, then there must exist a $j<k$ such 
that $\psi_{j}$ is of the form $\forall x \varphi$ and if $\psi_{k}$ is a formula that contains a primitive proposition $q \in L^{\prime \prime}$, then there must exist a $j<k$ such that $\psi_{j}$ is of the form $\neg A_{i} q$. The construction continues exactly as in the proof of Lemma A.4, where we take $\Delta_{0}=\Gamma / X_{i} \cup\left\{\neg \varphi, \neg \forall x A_{i} x\right\}$. Note that by construction, if $\psi_{j}=\neg A_{i} q$ for some $q \in L^{\prime \prime}$, then $q$ does not occur in $\Delta_{j-1}^{\prime}$. We claim

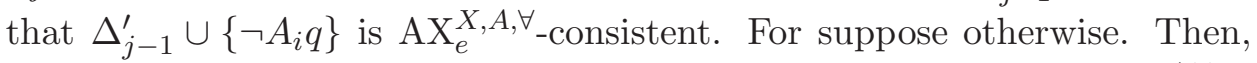
as above, there exists a subset $\left\{\beta_{1}, \ldots, \beta_{n}\right\} \subseteq \Delta_{j-1}^{\prime}$ such that $\operatorname{AX}_{e}^{X, A, \forall} \vdash$ $\beta \Rightarrow \forall x A_{i} x$ Since $\left\{\beta_{1}, \ldots, \beta_{n}, \neg \forall x A_{i} x\right\} \subseteq \Delta_{j-1}^{\prime}$, it follows that $\Delta_{j-1}^{\prime}$ is not $\mathrm{AX}_{e}^{X, A, \forall}$-consistent, a contradiction.

Therefore, $\Delta$ is a set of sentences that is acceptable and maximal $\mathrm{AX}_{e}^{X, A, \forall}$ consistent with respect to $L^{\prime}$ and includes $\Gamma / X_{i} \cup \neg \varphi \cup\left\{\neg A_{i} q: q \in L^{\prime \prime}\right\}$. The proof that $A_{i} \psi \in \Gamma$ implies $A_{i} \psi \in \Delta$ is identical to the first case. For the converse, suppose that $A_{i} \psi \in \Delta$. Then, by AGPP, $A_{i} q \in \Delta$ for all primitive propositions $q$ that appear in $\psi$. The construction of $\Delta$ guarantees that, for all primitive propositions in $L^{\prime}$, we have $A_{i} q \in \Delta$ iff $A_{i} q \in \Gamma$. Since $\Gamma$ is maximal $\mathbf{X}_{\mathbf{n}}^{\forall}$-consistent with respect to $L$, AGPP implies that $A_{i} \psi \in \Gamma$.

Lemma A.7 If $\varphi$ is a $\mathrm{AX}_{e}^{X, A, \forall}$-consistent sentence, then $\varphi$ is satisfiable in $\mathcal{N}_{n}^{a g p p, k a, \emptyset}(\Phi, \mathcal{X})$.

Proof: As usual, we construct a canonical model where the worlds are maximal consistent sets of formulas. However, now the worlds must also explicitly include the language. For technical reasons, we also assume that the language is infinite and co-infinite.

Let $M^{c}=\left(S, \mathcal{L}, \mathcal{K}_{1}, \ldots, \mathcal{K}_{n}, \mathcal{A}_{1}, \ldots, \mathcal{A}_{n}, \pi\right)$ be a canonical extended awareness structure constructed as follows

- $S=\left\{\left(s_{V}, L\right): V\right.$ is a set of sentences that is acceptable and maximal $\mathrm{AX}_{e}^{X, A, \forall}$-consistent with respect to $L$, where $L \subseteq \Phi$ is infinite and co-infinite\};

- $\mathcal{L}\left(\left(s_{V}, L\right)\right)=L$

- $\pi\left(\left(s_{V}, L\right), p\right)= \begin{cases}\text { true } & \text { if } p \in V, \\ \text { false } & \text { if } p \in(L-V) ;\end{cases}$

- $\mathcal{A}_{i}\left(\left(s_{V}, L\right)\right)=\left\{\varphi: A_{i} \varphi \in V\right\}$

- $\mathcal{K}_{i}\left(\left(s_{V}, L\right)\right)=\left\{\left(s_{W}, L^{\prime}\right): V / X_{i} \subseteq W\right.$ and $A_{i} \varphi \in W$ iff $A_{i} \varphi \in V$ for all formulas $\left.\varphi\right\}$. 
We show that if $\psi \in \mathcal{L}_{n}^{\forall, X, A}(L, \mathcal{X})$ is a sentence, then

$$
\left(M^{c},\left(s_{V}, L\right)\right) \models \psi \text { iff } \psi \in V \text {. }
$$

Note that this claim suffices to prove Lemma A.7 since, for all $L \subseteq \Phi$ that is infinite and co-infinite, if $\varphi \in \mathcal{L}_{n}^{\forall, X, A}(L, \mathcal{X})$ is a $\mathrm{AX}_{e}^{X, A, \forall}$-consistent sentence, by Lemmas A.2 and A.4, it is contained in a set of sentences that

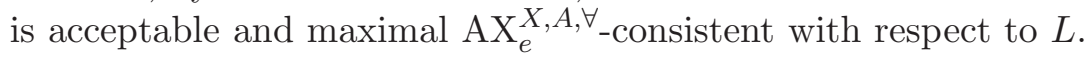

We prove (5) by induction of the depth of nesting of $\forall$, with a subinduction on the length of the sentence. The details are standard and left to the reader. For the case of $X_{i} \varphi$, we need Lemma A.6.

If $\varphi$ is consistent, by Lemmas A.2 and A.4, then $\varphi$ there is a set $L \subseteq \Phi$ that is infinite and co-infinite and contains $\Phi(\varphi)$ and a set $V$ of sentences

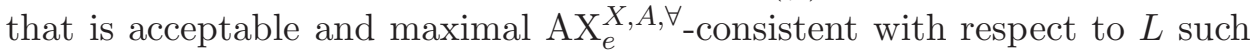
that $\varphi \in V$. By the argument above, $\left(M,\left(s_{V}, L\right)\right) \models \varphi$, showing that $\varphi$ is satisfiable, as desired.

To finish the completeness proof, suppose that $\varphi$ is valid in $\mathcal{N}_{n}^{a g p p, k a, \emptyset}(\Phi, \mathcal{X})$. Since $\varphi$ is a sentence, it follows that $\neg \varphi$ is a sentence and is not satisfiable in $\mathcal{N}_{n}^{a g p p, k a, \emptyset}(\Phi, \mathcal{X})$. So, by Lemma A.7, $\neg \varphi$ is not $\mathrm{AX}_{e}^{X, A,{ }^{\prime}}$-consistent. Thus, $\varphi$ is provable in $\operatorname{AX}_{e}^{X, A, \forall}$.

\section{Proposition 4.1:}

(a) $X A^{*}$ is valid in $\mathcal{N}_{n}^{t}(\Phi, \mathcal{X})$.

(b) Barcan* is valid in $\mathcal{N}_{n}^{r, e}(\Phi, \mathcal{X})$.

(c) $F A^{*}$ is valid in $\mathcal{N}_{n}^{r, e}(\Phi, \mathcal{X})$.

(d) $5^{*}$ is valid in $\mathcal{N}_{n}^{e}(\Phi, \mathcal{X})$.

Proof: For part (a), suppose that $(M, s) \models A_{i}^{*} \varphi$, where $M \in \mathcal{N}_{n}^{t}(\Phi, \mathcal{X})$. Thus, $(M, s) \models K_{i}(\varphi \vee \neg \varphi)$. Since the axiom 4 is valid in structures in $\mathcal{N}_{n}^{t}(\Phi, \mathcal{X})$, it follows that $(M, s) \models K_{i} K_{i}(\varphi \vee \neg \varphi)$, that is, $(M, s) \models K_{i} A_{i}^{*} \varphi$.

For part (b), suppose that $(M, s) \models A_{i}^{*}(\forall x \varphi) \wedge \forall x\left(A_{i}^{*} x \Rightarrow K_{i} \varphi\right)$, where $M \in \mathcal{N}_{n}^{r, e}(\Phi, \mathcal{X})$. It easily follows that $(M, s) \models A_{i}^{*}\left(\forall x A_{i}^{*} x \Rightarrow \forall x \varphi\right)$. Suppose, by way of contradiction, that $(M, s) \models \neg K_{i}\left(\forall x A_{i}^{*} x \Rightarrow \forall x \varphi\right)$. Then there must exist some world $t$ such that $(s, t) \in \mathcal{K}_{i}$ and $(M, t) \models \neg\left(\forall x A_{i}^{*} x \Rightarrow\right.$ $\forall x \varphi)$. Thus, $(M, t) \models \forall x A_{i}^{*} x$ and $(M, t) \models \neg \forall x \varphi$. Since $(M, t) \models \neg \forall x \varphi$, it follows that there exists $\psi \in \mathcal{L}_{n}^{K, X, A}(\mathcal{L}(t))$ such that $(M, t) \models \neg \varphi[x / \psi]$. Since $(M, t) \models \forall x A_{i}^{*} x$, we must have $(M, t) \models A_{i}^{*} \psi$. Thus, for every 
world $u$ such that $(t, u) \in \mathcal{K}_{i}$, it follows that $\psi \in \mathcal{L}_{n}^{K, X, A}(\mathcal{L}(u))$. Suppose that $(s, v) \in \mathcal{K}_{i}$. Since $\mathcal{K}_{i}$ is Euclidean and $(s, t) \in \mathcal{K}_{i}$, it follows that $(t, v) \in \mathcal{K}_{i}$ and, by the observation above, that $\psi \in \mathcal{L}_{n}^{K, X, A}(\mathcal{L}(v))$. Since $\mathcal{K}_{i}$ is reflexive and Euclidean, it follows that $(t, s) \in \mathcal{K}_{i}$, so the argument above also shows that $\psi \in \mathcal{L}_{n}^{K, X, A}(\mathcal{L}(s))$. Thus, $(M, s) \models A_{i}^{*} \psi$. Since $(M, s) \models \forall x\left(A_{i}^{*} x \Rightarrow K_{i} \varphi\right)$, it follows that $(M, s) \models K_{i} \varphi[x / \psi]$. Thus, $(M, t) \models \varphi[x / \psi]$, a contradiction.

Finally, for part (c), suppose that $(M, s) \models \forall x \neg A_{i}^{*} x$, where $M \in \mathcal{N}_{n}^{r, e}(\Phi, \mathcal{X})$. Thus, for every primitive proposition $p \in \mathcal{L}(s)$, there exists some $t_{p}$ such that $\left(s, t_{p}\right) \in \mathcal{K}_{i}$ and $p \notin \mathcal{L}\left(t_{p}\right)$. Let $u$ be an arbitrary world such that $(s, u) \in \mathcal{K}_{i}$. Let $\varphi$ be an arbitrary quantifier-free sentence in $\mathcal{L}_{n}^{\forall, K, X, A}(\mathcal{L}(u), \mathcal{X})$. If $\Phi(\varphi) \cap \mathcal{L}(s) \neq \emptyset$, suppose that $p \in \Phi(\varphi) \cap \mathcal{L}(s)$. By assumption, $p \notin$ $\mathcal{L}\left(t_{p}\right)$. Since $\mathcal{K}_{i}$ is Euclidean, $\left(u, t_{p}\right) \in \mathcal{K}_{i}$. Thus, $(M, u) \models \neg A_{i}^{*} \varphi$. If $\Phi(\varphi) \cap \mathcal{L}(s)=\emptyset$, note that since $\mathcal{K}_{i}$ is reflexive and Euclidean, the fact that $(s, s)$ and $(s, u)$ are in $\mathcal{K}_{i}$ implies that $(u, s) \in \mathcal{K}_{i}$. Hence, we again have that $(M, u) \models \neg A_{i}^{*} \varphi$.

The proof of part $(\mathrm{d})$ is standard, and left to the reader.

\section{Theorem 4.2:}

(a) $\mathrm{AX}_{e}^{K, A^{*}} \cup\left\{\mathrm{T}, 4,5^{*}\right\}$ is a sound and complete axiomatization of $\mathcal{L}_{n}^{K}(\Phi)$ with respect to $\mathcal{N}_{n}^{r, t, e}(\Phi)$.

(b) $\mathrm{AX}_{e}^{K, A^{*}, \forall} \cup\left\{\mathrm{T}, 4,5^{*}\right\}$ is a sound and complete axiomatization of the sentences in $\mathcal{L}_{n}^{\forall, K}(\Phi, \mathcal{X})$ with respect to $\mathcal{N}_{n}^{r, t, e}(\Phi, \mathcal{X})$.

(c) $\mathrm{AX}_{e}^{K, X, A, A^{*}, \forall} \cup\left\{\mathrm{T}, 4,5^{*}\right\}$ is a sound and complete axiomatization of the sentences in $\mathcal{L}_{n}^{\forall, K, X, A}(\Phi, \mathcal{X})$ with respect to $\mathcal{N}_{n}^{r, t, e}(\Phi, \mathcal{X})$.

Proof: The proof of part (c) is identical to the proof of Theorem 4.1, except that $X_{i}$ and $A_{i}$ are replaced by $K_{i}$ and $A_{i}^{*}$, respectively, and in Lemma A.7, another step is needed in the induction to deal with $X_{i}$ that uses the extra axiom A0 in the standard way.

For part (b), note that since $X_{i}$ and $A_{i}$ are not part of the language the axioms of $\mathrm{AX}_{e}^{K, X, A, A^{*}, \forall}$ that mention these operators are not needed in the induction of Lemma A.7. Therefore, the proof is the same.

The proof of part (a) is similar to that of Theorem 4.1, except that the following lemma is used instead of Lemma A.7.

Lemma A.8 If $\varphi$ is a $\mathrm{AX}_{e}^{K, A^{*}} \cup\left\{\mathrm{T}, 4,5^{*}\right\}$-consistent sentence in $\mathcal{L}_{n}^{K}(\Phi)$, then $\varphi$ is satisfiable in $\mathcal{N}_{n}^{r, t, e}(\Phi)$. 
Proof: Let $M^{c}=\left(S, \mathcal{L}, \mathcal{K}_{1}, \ldots, \mathcal{K}_{n}, \mathcal{A}_{1}, \ldots, \mathcal{A}_{n}, \pi\right)$ be a canonical extended awareness structure constructed as follows

- $S=\left\{\left(s_{V}, L\right): V\right.$ is a set of sentences in $\mathcal{L}_{n}^{K}(L)$ that is maximal $\mathrm{AX}_{e}^{K, A^{*}} \cup\left\{\mathrm{T}, 4,5^{*}\right\}$-consistent with respect to $L$ and $\left.L \subseteq \Phi\right\}$;

- $\mathcal{L}\left(\left(s_{V}, L\right)\right)=L$;

- $\pi\left(\left(s_{V}, L\right), p\right)= \begin{cases}\text { true } & \text { if } p \in V, \\ \text { false } & \text { if } p \in(L-V)\end{cases}$

- $\mathcal{A}_{i}\left(\left(s_{V}, L\right)\right)$ is arbitrary;

- $\mathcal{K}_{i}\left(\left(s_{V}, L\right)\right)=\left\{\left(s_{W}, L\right): V / K_{i} \subseteq W\right\}$.

It is easy to see that $M^{c} \in \mathcal{N}_{n}^{r, t, e}(\Phi)$. As usual, to prove Lemma A.8, we now show that for every $\psi \in \mathcal{L}_{n}^{K}(L)$,

$$
\left(M^{c},\left(s_{V}, L\right)\right) \models \psi \text { iff } \psi \in V .
$$

We prove (6) by induction on the length of the formula. All the cases are standard, except for the case that $\psi=K_{i} \psi^{\prime}$. In this case, if $\psi \in$ $V$, then $\psi^{\prime} \in W$ for every $W$ such that $\left(s_{W}, L^{\prime}\right) \in \mathcal{K}_{i}\left(\left(s_{V}, L\right)\right)$. By the induction hypothesis, $\left(M^{c},\left(s_{W}, L^{\prime}\right)\right) \models \psi^{\prime}$ for every $\left(s_{W}, L^{\prime}\right) \in \mathcal{K}_{i}\left(\left(s_{V}, L\right)\right)$, so $\left(M^{c},\left(s_{V}, L\right)\right) \models K_{i} \psi^{\prime}$.

If $\psi \notin V$, since $\psi \in \mathcal{L}_{n}^{K}(L)$, it follows that $\neg \psi \in V$. If $A_{i}^{*} \psi^{\prime} \notin V$, then $\psi^{\prime}$ is not defined at some world $\left(s_{W}, L^{\prime}\right) \in \mathcal{K}_{i}\left(\left(s_{V}, L\right)\right)$ which implies that $\left(M^{c},\left(s_{V}, L\right)\right) \forall \forall \psi$. If $A_{i}^{*} \psi^{\prime} \in V$, then we need to show that $V / K_{i} \cup\left\{\neg \psi^{\prime}\right\}$ is $\operatorname{AX}_{e}^{K, A^{*}} \cup\left\{\mathrm{T}, 4,5^{*}\right\}$-consistent. Suppose not. Then there exists a subset $\left\{\beta_{1}, \ldots, \beta_{k}\right\} \subseteq V / K_{i}$ such that

$$
\mathrm{AX}_{e}^{K, A^{*}} \cup\left\{\mathrm{T}, 4,5^{*}\right\} \vdash \beta \Rightarrow \psi^{\prime},
$$

where $\beta=\beta_{1} \wedge \cdots \wedge \beta_{k}$. By Gen*, it follows that

$$
\mathrm{AX}_{e}^{K, A^{*}} \cup\left\{\mathrm{T}, 4,5^{*}\right\} \vdash \mathrm{A}_{\mathrm{i}}^{*}\left(\beta \Rightarrow \psi^{\prime}\right) \Rightarrow \mathrm{K}_{\mathrm{i}}\left(\beta \Rightarrow \psi^{\prime}\right) .
$$

Since $\left\{\beta_{1}, \ldots, \beta_{k}\right\} \subseteq V / K_{i}$, it follows that $\left\{K_{i} \beta_{1}, \ldots, K_{i} \beta_{k}\right\} \subseteq V$. Thus, by A0*, we have $\left\{A_{i}^{*} \beta_{1}, \ldots, A_{i}^{*} \beta_{k}\right\} \subseteq V$. Thus, $A_{i}^{*}\left(\beta \Rightarrow \psi^{\prime}\right) \in V$ and $K_{i} \beta \in V$. Therefore, $K_{i} \psi^{\prime} \in V$, a contradiction.

Since $V / K_{i} \cup\left\{\neg \psi^{\prime}\right\} \subseteq \mathcal{L}_{n}^{K}(L)$ and is $\mathrm{AX}_{e}^{K, A^{*}} \cup\left\{\mathrm{T}, 4,5^{*}\right\}$-consistent, it follows that there exists a set of sentences $W$ that is maximal $\operatorname{AX}_{e}^{K, A^{*}} \cup$ $\left\{\mathrm{T}, 4,5^{*}\right\}$-consistent with respect to $L$ and contains $V / K_{i} \cup\left\{\neg \psi^{\prime}\right\}$. Thus, 
$\left(s_{W}, L\right) \in \mathcal{K}_{i}\left(\left(s_{V}, L\right)\right)$ and, by the induction hypothesis, $\left(M^{c},\left(s_{W}, L\right)\right) \not \forall \psi^{\prime}$. Thus, $\left(M^{c},\left(s_{V}, L\right)\right) \not \models \psi$.

Proposition 5.3: $\quad$ Let $M$ be an extended awareness structure satisfying agpp and $k a$.

(a) $(M, s) \models X_{i} \varphi \vee X_{i} \neg X_{i} \varphi$ iff $(M, s) \models A_{i} \varphi \wedge\left(K_{i} \varphi \vee K_{i} \neg K_{i} \varphi\right)$. Moreover, if $M$ is Euclidean, then $(M, s) \models X_{i} \varphi \vee X_{i} \neg X_{i} \varphi$ iff $(M, s) \models A_{i} \varphi$.

(b) $(M, s) \models X_{i}(\varphi \vee \neg \varphi)$ iff $(M, s) \models A_{i} \varphi$.

Proof: For part (a), first suppose that $(M, s) \models X_{i} \varphi \vee X_{i} \neg X_{i} \varphi$. Thus, either (i) $(M, s) \models X_{i} \varphi$ or (ii) $(M, s) \models X_{i} \neg X_{i} \varphi$. In case (i), it follows that $(M, s) \models A_{i} \varphi$ and $(M, s) \models K_{i} \varphi$. Thus, $(M, s) \models A_{i} \varphi \wedge\left(K_{i} \varphi \vee K_{i} \neg K_{i} \varphi\right)$. In case (ii), it follows that $(M, s) \models A_{i} \neg X_{i} \varphi$ and $(M, s) \models K_{i} \neg X_{i} \varphi$. By agpp, we have $(M, s) \models A_{i} \varphi$. Let $t \in \mathcal{K}_{i}(s)$. By $k a$, we have $(M, t) \models A_{i} \varphi$. And as $(M, s) \models K_{i} \neg X_{i} \varphi$, it follows that $(M, t) \models \neg X_{i} \varphi$. Thus, $(M, t) \models \neg K_{i} \varphi$, and so $(M, s) \models K_{i} \neg K_{i} \varphi$. For the other direction, suppose that $(M, s) \models$ $A_{i} \varphi \wedge\left(K_{i} \varphi \vee K_{i} \neg K_{i} \varphi\right)$. Thus, either (i) $(M, s) \models A_{i} \varphi \wedge K_{i} \varphi$ or (ii) $(M, s) \models$ $A_{i} \varphi \wedge K_{i} \neg K_{i} \varphi$. In case (i), it follows that $(M, s) \models X_{i} \varphi$. In case (ii), agpp implies that $(M, s) \models A_{i} \neg X_{i} \varphi$. Let $t \in \mathcal{K}_{i}(s)$. Thus, $(M, t) \models \neg K_{i} \varphi$, which implies that $(M, t) \models \neg X_{i} \varphi$. Therefore, $(M, s) \models X_{i} \neg X_{i} \varphi$.

Now suppose that $M$ is Euclidean. Recall that by $k a$ it follows that $(M, s) \models A_{i} \varphi$ implies $(M, s) \models A_{i}^{*} \varphi$. Proposition 5.1 shows that, if $M$ is Euclidean, then $(M, s) \models A_{i}^{*} \varphi$ implies $(M, s) \models A_{i}^{\prime} \varphi$. Combining both results, we get $(M, s) \models A_{i} \varphi$ implies $(M, s) \models K_{i} \varphi \vee K_{i} \neg K_{i} \varphi$.

For part (b), note that by agpp and the semantics of $X_{i} \varphi$, it follows that $(M, s) \models X_{i}(\varphi \vee \neg \varphi)$ implies $(M, s) \models A_{i} \varphi$. For the converse, recall that $(M, s) \models A_{i} \varphi$ implies $(M, s) \models A_{i}^{*} \varphi$. By agpp and the definition of $(M, s) \models X_{i} \varphi,(M, s) \models A_{i} \varphi \wedge A_{i}^{*} \varphi$ implies $(M, s) \models X_{i}(\varphi \vee \neg \varphi)$, as desired.

\section{Acknowledgments}

The first author was supported in part by NSF under grants ITR-0325453, IIS-0534064, IIS-0812045, and IIS-0911036, by AFOSR under grants FA955005-1-0055, FA9550-08-1-0438, and FA9550-09-1-0266, and by ARO under grant W911NF-09-1-0281. The second author is supported in part by FACEPE under grants APQ-0150-1.02/06 and APQ-0219-3.08/08, and by MCT/CNPq under grants 475634/2007-1 and 306358/2010-7. 


\section{References}

Ågotnes, T., Alechina, N., 2007. Full and relative awareness: a decidable logic for reasoning about knowledge of unawareness. In: Theoretical Aspects of Rationality and Knowledge: Proc. Eleventh Conference (TARK 2007). pp. 6-14.

Board, O., Chung, K.-S., 2009. Object-based unawareness: theory and applications. Working Paper 378, University of Pittsburgh.

Board, O., Chung, K.-S., Schipper, B. C., 2011. Two models of unawareness: Comparing the object-based and the subjective-state-space approaches. Synthese 179, 13-34.

Bull, R., 1969. On modal logic with propositional quantifiers. Journal of Symbolic Logic 34, 257-263.

Dekel, E., Lipman, B., Rustichini, A., 1998. Standard state-space models preclude unawareness. Econometrica 66, 159-173.

Engelhardt, K., Meyden, R. v. d., Moses, Y., 1998. Knowledge and the logic of local propositions. In: Theoretical Aspects of Rationality and Knowledge: Proc. Seventh Conference (TARK 1998). pp. 29-41.

Fagin, R., Halpern, J. Y., 1988. Belief, awareness, and limited reasoning. Artificial Intelligence 34, 39-76.

Fagin, R., Halpern, J. Y., Moses, Y., Vardi, M. Y., 1995. Reasoning About Knowledge. MIT Press, Cambridge, Mass., a slightly revised paperback version was published in 2003.

Fagin, R., Halpern, J. Y., Vardi, M. Y., 1992. What can machines know? On the properties of knowledge in distributed systems. Journal of the ACM $39(2), 328-376$.

Feinberg, Y., 2011. Strategic communication. In: Theoretical Aspects of Rationality and Knowledge: Proc. Thirteenth Conference (TARK 2011). pp. $1-11$.

Fine, K., 1970. Propositional quantifiers in modal logic. Theoria 36, 336-346.

Halpern, J. Y., 2001. Alternative semantics for unawareness. Games and Economic Behavior 37, 321-339. 
Halpern, J. Y., Rêgo, L. C., 2008. Interactive unawareness revisited. Games and Economic Behavior 62 (1), 232-262.

Halpern, J. Y., Rêgo, L. C., 2009. Reasoning about knowledge of unawareness. Games and Economic Behavior 67 (2), 503-525.

Halpern, J. Y., Rêgo, L. C., 2013. Extensive games with possibly unaware players. Mathematical Social SciencesTo appear.

Heifetz, A., Meier, M., Schipper, B., 2006. Interactive unawareness. Journal of Economic Theory 130, 78-94.

Heifetz, A., Meier, M., Schipper, B., 2008. A canonical model for interactive unawareness. Games and Economic Behavior 62, 305-324.

Hintikka, J., 1962. Knowledge and Belief. Cornell University Press, Ithaca, N.Y.

Hughes, G. E., Cresswell, M. J., 1996. A New Introduction to Modal Logic. Routledge, London.

Kaplan, D., 1970. S5 with quantifiable propositional variables. Journal of Symbolic Logic 35, 355.

Kripke, S., 1959. A completeness theorem in modal logic. Journal of Symbolic Logic 24, 1-14.

Modica, S., Rustichini, A., 1994. Awareness and partitional information structures. Theory and Decision 37, 107-124.

Modica, S., Rustichini, A., 1999. Unawareness and partitional information structures. Games and Economic Behavior 27 (2), 265-298.

Schipper, B., 2010. Awareness-dependent subjective expected utility, unpublished manuscript.

Sillari, G., 2008a. Models of awareness. In: Logic and the Foundations of Game and Decision Theory. Texts in Logic and Games, Vol. 2. Amsterdam University Press.

Sillari, G., 2008b. Quantified logic of awareness and impossible possible worlds. Review of Symbolic Logic 1 (4), 514-529.

Walker, O., 2011. Unawareness with "possible" possible worlds, manuscript. 